\title{
Nonlocal boundary value problems for integro-differential Langevin equation via the generalized Caputo proportional fractional derivative
}

\author{
Bounmy Khaminsou', Chatthai Thaiprayoon', Jehad Alzabut ${ }^{2}$ and Weerawat Sudsutad ${ }^{3 *}$ (ID
}

\author{
${ }^{*}$ Correspondence: \\ weerawat@nmu.ac.th \\ ${ }^{3}$ Department of General Education, \\ Faculty of Science and Health \\ Technology, Navamindradhiraj \\ University, Bangkok, 10300, Thailand \\ Full list of author information is \\ available at the end of the article
}

\begin{abstract}
Results reported in this paper study the existence and stability of a class of implicit generalized proportional fractional integro-differential Langevin equations with nonlocal fractional integral conditions. The main theorems are proved with the help of Banach's, Krasnoselskii's, and Schaefer's fixed point theorems and Ulam's approach. Finally, an example is given to demonstrate the applicability of our theoretical findings.

MSC: 34A08; 34B10; 34D20

Keywords: Fractional Langevin equation; Generalized proportional fractional derivative; Ulam stability; Existence and uniqueness; Nonlocal integral conditions
\end{abstract}

\section{Introduction}

Fractional calculus is a mathematical branch investigating the properties dealing with arbitrary order differential and integral operators. Fractional differential equation have been an excellent instrument in the mathematical modeling of dynamical systems and real world problems, such as physics, biological and chemical engineering, aerodynamics, earthquake vibrations, fractals and chaotic, nonlinear control theory, signal and image processing, artificial intelligence, etc. However, many researchers introduced various definitions of fractional derivative and integral operators of arbitrary order. For more details, we refer the reader to the books [1-5]. Some recent contributions to the theory of fractional differential equations and its applications can be seen in [6-9] and the references cited therein.

In this paper, we study the existence and stability of solutions for the following generalized proportional fractional (GPF) functional integro-differential Langevin equation with

(c) The Author(s) 2020. This article is licensed under a Creative Commons Attribution 4.0 International License, which permits use, sharing, adaptation, distribution and reproduction in any medium or format, as long as you give appropriate credit to the original author(s) and the source, provide a link to the Creative Commons licence, and indicate if changes were made. The images or other third party material in this article are included in the article's Creative Commons licence, unless indicated otherwise in a credit line to the material. If material is not included in the article's Creative Commons licence and your intended use is not permitted by statutory regulation or exceeds the permitted use, you will need to obtain permission directly from the copyright holder. To view a copy of this licence, visit http://creativecommons.org/licenses/by/4.0/. 
variable coefficient and nonlocal fractional integral conditions:

$$
\left\{\begin{array}{l}
{ }_{a}^{C} D^{q_{1}, \rho}\left({ }_{a}^{C} D^{q_{2}, \rho}+\lambda(t)\right) x(t)=f(t, x(t), x(\theta(t)),(\mathcal{K} x)(t)), \quad t \in[a, T] \\
\sum_{i=1}^{m} \kappa_{i a} I^{\mu_{i}, \rho} x\left(\sigma_{i}\right)=\sum_{j=1}^{n} \alpha_{j a} I^{\beta_{j}, \rho} x\left(\eta_{j}\right), \\
\sum_{k=1}^{p} \omega_{k a} I^{\gamma_{k}, \rho} x\left(\psi_{k}\right)=\sum_{l=1}^{r} v_{l a} I^{\varphi_{l}, \rho} x\left(\xi_{l}\right),
\end{array}\right.
$$

where ${ }_{a}^{C} D^{q, \rho}$ denotes the GPF derivative of order $q, q=\left\{q_{1}, q_{2}\right\}$ with $0<q_{1}, q_{2} \leq 1$, $1<q_{1}+q_{2} \leq 2, \rho>0$, in Caputo type, ${ }_{a} I^{w, \rho}$ denotes the GPF integral of order $w>0$, $w=\left\{\mu_{i}, \beta_{j}, \gamma_{k}, \varphi_{l}\right\}, \rho>0, \kappa_{i}, \alpha_{j}, \omega_{k}, v_{l} \in \mathbb{R}, \sigma_{i}, \eta_{j}, \psi_{k}, \xi_{l} \in(a, T)$ for all $i=1,2, \ldots, m, j=$ $1,2, \ldots, n, k=1,2, \ldots, p, l=1,2, \ldots, r, m, n, p, r \in \mathbb{N}, \lambda \in C([a, T], \mathbb{R}), f \in C\left([a, T] \times \mathbb{R}^{3}, \mathbb{R}\right)$, $\theta \in C([a, T],[a, T])$, and

$$
(\mathcal{K} x)(t)=\int_{a}^{t} \phi(t, s, x(s)) d s, \quad t \in[a, T]
$$

where $\phi \in C\left([a, T]^{2} \times \mathbb{R},[a, \infty)\right)$.

The Langevin equation has been used to describe the dynamics of physical phenomena in the fluctuating environment of mathematical physics [10,11]. For a system in complex phenomena, it has been realized that the integer order of the Langevin equation does not provide the accurate representation of dynamical systems. Therefore, one of the best ways to overcome this disadvantage is to replace the integer order derivative by the fractional order derivative [12-15]. The popular research interest in fractional Langevin equations is focused on the investigation of existence and stability of solutions. In this context, the literature has witnessed the appearance of many results on Langevin equations within various types of fractional operators and using different techniques, we refer the reader to the papers [16-29] and the references therein. It is worth mentioning here that all of the above cited work has been conducted in the frame of the classical Riemann-Liouville, Caputo, and Hadamard fractional operators. Further, the problem of Langevin has been considered using some generalized fractional derivatives in which, for instance, Atangana-Baleanu and Hilfer fractional derivatives were employed [30, 31].

Inspired by the above work and with the hope of considering generalized fractional derivative that includes the classical derivatives as particular cases, we accommodate the newly defined GPF derivative to study the problem of Langevin equation. The new derivative $D^{p, q} x(t)$ involves two parameters and has the features that the semigroup property is preserved, nonlocal character is possessed, and upon limiting cases it converges to the original function and its derivative. The GPF derivative is well behaved and has a substantial advantage over the classical derivatives in the sense that it generalizes previously defined derivatives in the literature. We list here some recent results which have been elaborated in the frame of GPF derivative [32-37]. Exploring the literature and in view of equations considered in the aforesaid references, one can figure out that equation (1.1) is entirely different from the equations investigated earlier. The nonlinearity function incorporates an integral term, (1.1) includes variable coefficient, and the boundary conditions are formulated in general settings.

The manuscript is processed as follows. Sect. 2 is essential in its nature as it presents preliminary definitions and results. In Sect. 3, we establish some appropriate conditions for the existence and uniqueness of solutions of problem (1.1) via the technique of fixed 
point theorems. In Sect. 4, we set up applicable results under which the solution of problem (1.1) fulfills the conditions of different kinds of Ulam stability. The validity of discussed results is illustrated by a particular example in Sect. 5 . We end the paper by a conclusion.

\section{Preliminaries}

This section presents some fundamental definitions and lemmas that will be used in this paper. For interpretations and proofs, the reader can consult the papers [38-40].

Throughout this paper, we define $\mathbb{E}=C([a, T], \mathbb{R})$ as the Banach space of all continuous functions from $[a, T]$ into $\mathbb{R}$ equipped with the norm $\|x\|_{\mathbb{E}}=\sup _{t \in[a, T]}\{|x(t)|\}$.

Definition 2.1 ([38]) For $\rho \in(0,1]$ and $\alpha \in \mathbb{R}^{+}$, the generalized proportional fractional (GPF) integral of function $f$ of order $\alpha$ is defined by

$$
\left({ }_{a} I^{\alpha, \rho} f\right)(t)=\frac{1}{\rho^{\alpha} \Gamma(\alpha)} \int_{a}^{t} e^{\frac{\rho-1}{\rho}(t-s)}(t-s)^{\alpha-1} f(s) d s,
$$

where $\Gamma(\cdot)$ represents the gamma function.

Definition 2.2 ([38]) For $\rho \in(0,1]$ and $\alpha \in \mathbb{R}^{+}$, the generalized proportional fractional (GPF) derivative of Caputo type of function $f$ of order $\alpha$ is defined by

$$
\left({ }_{a}^{C} D^{\alpha, \rho} f\right)(t)=\frac{1}{\rho^{n-\alpha} \Gamma(n-\alpha)} \int_{a}^{t} e^{\frac{\rho-1}{\rho}(t-\tau)}(t-\tau)^{n-\alpha-1} D^{n, \rho} f(\tau) d \tau,
$$

where $n=[\alpha]+1$ with $[\alpha]$ represents the integer part of the real number $\alpha$ and $\left(D^{n, \rho} f\right)(t)=$ $\left(D^{\rho} f(t)\right)^{n}$ with $\left(D^{\rho} f\right)(t)=(1-\rho) f(t)-\rho f^{\prime}(t)$. Note that $\lim _{\rho \rightarrow 0}\left(D^{\rho} f\right)(t)=f(t)$.

Lemma 2.3 ([38]) For $\rho \in(0,1]$ and $n=[\alpha]+1$, we have $\left({ }_{a}^{C} D^{\alpha, \rho}{ }_{a} I^{\alpha, \rho} f\right)(t)=f(t)$, and

$$
\left({ }_{a} I_{a}^{\alpha, \rho C} D^{\alpha, \rho} f\right)(t)=f(t)-e^{\frac{\rho-1}{\rho}(t-a)} \sum_{k=0}^{n-1} \frac{D^{k, \rho} f(a)}{\rho^{k} k !}(t-a)^{k} .
$$

Proposition 2.4 ([38]) Let $\alpha \geq 0$ and $\beta>0$. Then, for any $\rho \in(0,1]$ and $n=[\alpha]+1$, we have

(i) $\left({ }_{a} I^{\alpha, \rho} e^{\frac{\rho-1}{\rho} s}(s-a)^{\beta-1}\right)(t)=\frac{\Gamma(\beta)}{\Gamma(\beta+\alpha) \rho^{\alpha}} e^{\frac{\rho-1}{\rho} t}(t-a)^{\beta+\alpha-1}, \alpha>0$.

(ii) $\left({ }_{a}^{C} D^{\alpha, \rho} e^{\frac{\rho-1}{\rho} s}(s-a)^{\beta-1}\right)(t)=\frac{\rho^{\alpha} \Gamma(\beta)}{\Gamma(\beta-\alpha)} e^{\frac{\rho-1}{\rho} x}(t-a)^{\beta-\alpha-1}, \alpha>n$.

(iii) $\left({ }_{a}^{C} D^{\alpha, \rho} e^{\frac{\rho-1}{\rho} s}(s-a)^{k}\right)(t)=0, \alpha>n, k=0,1, \ldots, n-1$.

Fixed point theorems play a major role in establishing the existence theory for problem (1.1). We collect here some well-known fixed point theorems for the sake of completeness.

Lemma 2.5 (Banach's fixed point theorem [40]) Let D be a nonempty closed subset of a Banach space $E$. Then any contraction mapping $T$ from $D$ into itself has a unique fixed point.

Lemma 2.6 (Krasnoselskii's fixed point theorem [41]) Let $M$ be a closed, bounded, convex, and nonempty subset of a Banach space X. Let $A, B$ be the operators such that (i) $A x+B y \in$ $M$ whenever $x, y \in M$; (ii) $A$ is compact and continuous; (iii) $B$ is a contraction mapping. Then there exists $z \in M$ such that $z=A z+B z$. 
Lemma 2.7 (Schaefer's fixed point theorem [40]) Let $\mathbb{M}$ be a Banach space and $T: \mathbb{M} \rightarrow$ $\mathbb{M}$ be a completely continuous operator, and let the set $D=\{x \in \mathbb{M}: x=\kappa T x, 0<\kappa \leq 1\}$ be bounded. Then $T$ has a fixed point in $\mathbb{M}$.

In order to transform the main problem into a fixed point problem, (1.1) must be converted to an equivalent Volterra integral equation. We provide the following lemma, which is important in our main results.

Lemma 2.8 Let $h:[a, T] \rightarrow \mathbb{R}$ be a continuous function, $0<q_{1}, q_{2} \leq 1,1<q_{1}+q_{2} \leq 2$ and $\rho, \mu_{i}, \beta_{j}, \gamma_{k}, \varphi_{l},>0, \kappa_{i}, \alpha_{j}, \omega_{k}, v_{l} \in \mathbb{R}$ and $\sigma_{i}, \eta_{j}, \psi_{k}, \xi_{l} \in(a, T)$ for all $i=1,2, \ldots, m$, $j=1,2, \ldots, n, k=1,2, \ldots, p, l=1,2, \ldots, r, m, n, p, r \in \mathbb{N}$. Then the function $x \in \mathbb{E}$ is the solution to the following linear GPF Langevin equation equipped with nonlocal fractional integral conditions:

$$
\left\{\begin{array}{l}
{ }_{a}^{C} D^{q_{1}, \rho}\left({ }_{a}^{C} D^{q_{2}, \rho}+\lambda(t)\right) x(t)=h(t), \quad t \in[a, T], \\
\sum_{i=1}^{m} \kappa_{i a} I^{\mu_{i}, \rho} x\left(\sigma_{i}\right)=\sum_{j=1}^{n} \alpha_{j a} I^{\beta_{j}, \rho} x\left(\eta_{j}\right), \\
\sum_{k=1}^{p} \omega_{k a} I^{\gamma_{k}, \rho} x\left(\psi_{k}\right)=\sum_{l=1}^{r} v_{l a} I^{\varphi, \rho} x\left(\xi_{l}\right),
\end{array}\right.
$$

if and only if $x$ satisfies the following fractional integral equation:

$$
\begin{aligned}
& x(t)={ }_{a} I^{q_{1}+q_{2}, \rho} h(t)-{ }_{a} I^{q_{2}, \rho} \lambda(t) x(t) \\
& +\frac{e^{\frac{\rho-1}{\rho}(t-a)}}{\Omega}\left[( \frac { \Omega _ { 4 } ( t - a ) ^ { q _ { 2 } } } { \rho ^ { q _ { 2 } } \Gamma ( q _ { 2 } + 1 ) } - \Omega _ { 3 } ) \left(\sum_{j=1}^{n} \alpha_{j}\left[I_{a} I^{q_{1}+q_{2}+\beta_{j}, \rho} h\left(\eta_{j}\right)-{ }_{a} I^{q_{2}+\beta_{j}, \rho} \lambda\left(\eta_{j}\right) x\left(\eta_{j}\right)\right]\right.\right. \\
& \left.-\sum_{i=1}^{m} \kappa_{i}\left[{ }_{a} I^{q_{1}+q_{2}+\mu_{i}, \rho} h\left(\sigma_{i}\right)-{ }_{a} I^{q_{2}+\mu_{i}, \rho} \lambda\left(\sigma_{i}\right) x\left(\sigma_{i}\right)\right]\right) \\
& +\left(\Omega_{1}-\frac{\Omega_{2}(t-a)^{q_{2}}}{\rho^{q_{2}} \Gamma\left(q_{2}+1\right)}\right)\left(\sum_{l=1}^{r} v_{l}\left[I_{a} I^{q_{1}+q_{2}+\varphi_{l}, \rho} h\left(\xi_{l}\right)-{ }_{a} I^{q_{2}+\varphi_{l}, \rho} \lambda\left(\xi_{l}\right) x\left(\xi_{l}\right)\right]\right. \\
& \left.\left.-\sum_{k=1}^{p} \omega_{k}\left[I^{q_{1}+q_{2}+\gamma_{k}, \rho} h\left(\psi_{k}\right)-{ }_{a} I^{q_{2}+\gamma_{k}, \rho} \lambda\left(\psi_{k}\right) x\left(\psi_{k}\right)\right]\right)\right] \text {, }
\end{aligned}
$$

where

$$
\begin{aligned}
& \Omega_{1}=\sum_{i=1}^{m} \frac{\kappa_{i}\left(\sigma_{i}-a\right)^{q_{2}+\mu_{i}} e^{\frac{\rho-1}{\rho}\left(\sigma_{i}-a\right)}}{\rho^{q_{2}+\mu_{i}} \Gamma\left(q_{2}+\mu_{i}+1\right)}-\sum_{j=1}^{n} \frac{\alpha_{j}\left(\eta_{j}-a\right)^{q_{2}+\beta_{j}} e^{\frac{\rho-1}{\rho}\left(\eta_{j}-a\right)}}{\rho^{q_{2}+\beta_{j}} \Gamma\left(q_{2}+\beta_{j}+1\right)}, \\
& \Omega_{2}=\sum_{i=1}^{m} \frac{\kappa_{i}\left(\sigma_{i}-a\right)^{\mu_{i}} e^{\frac{\rho-1}{\rho}\left(\sigma_{i}-a\right)}}{\rho^{\mu_{i}} \Gamma\left(\mu_{i}+1\right)}-\sum_{j=1}^{n} \frac{\alpha_{j}\left(\eta_{j}-a\right)^{\beta_{j}} e^{\frac{\rho-1}{\rho}\left(\eta_{j}-a\right)}}{\rho^{\beta_{j}} \Gamma\left(\beta_{j}+1\right)}, \\
& \Omega_{3}=\sum_{k=1}^{p} \frac{\omega_{k}\left(\psi_{k}-a\right)^{q_{2}+\gamma_{k}} e^{\frac{\rho-1}{\rho}\left(\psi_{k}-a\right)}}{\rho^{q_{2}+\gamma_{k}} \Gamma\left(q_{2}+\gamma_{k}+1\right)}-\sum_{l=1}^{r} \frac{v_{l}\left(\xi_{l}-a\right)^{q_{2}+\varphi_{l}} e^{\frac{\rho-1}{\rho}\left(\xi_{l}-a\right)}}{\rho^{q_{2}+\varphi_{l}} \Gamma\left(q_{2}+\varphi_{l}+1\right)}, \\
& \Omega_{4}=\sum_{k=1}^{p} \frac{\omega_{k}\left(\psi_{k}-a\right)_{k}^{\gamma} e^{\frac{\rho-1}{\rho}\left(\psi_{k}-a\right)}}{\rho^{\gamma_{k}} \Gamma\left(\gamma_{k}+1\right)}-\sum_{l=1}^{r} \frac{v_{l}\left(\xi_{l}-a\right)^{\varphi_{l}} e^{\frac{\rho-1}{\rho}\left(\xi_{l}-a\right)}}{\rho^{\varphi_{l} \Gamma\left(\varphi_{l}+1\right)},} \\
& \Omega=\Omega_{1} \Omega_{4}-\Omega_{2} \Omega_{3} \neq 0 .
\end{aligned}
$$


Proof Let $x$ be a solution of problem (2.4). By using Lemma 2.3 with Proposition 2.4 (i), the first equation of (2.4) can be written as an equivalent integral equation

$$
x(t)={ }_{a} I^{q_{1}+q_{2}, \rho} h(t)-{ }_{a} I^{q_{2}, \rho} \lambda(t) x(t)+c_{1} \frac{(t-a)^{q_{2}} e^{\frac{\rho-1}{\rho}(t-a)}}{\rho^{q_{2}} \Gamma\left(q_{2}+1\right)}+c_{2} e^{\frac{\rho-1}{\rho}(t-a)},
$$

where arbitrary constants $c_{1}, c_{2} \in \mathbb{R}$.

Taking the GPF integral operator ${ }_{a} I^{w, \rho}$ into (2.11), we obtain

$$
\begin{aligned}
{ }_{a} I^{w, \rho} x(t)= & { }_{a} I^{q_{1}+q_{2}+w, \rho} h(t)-{ }_{a} I^{q_{2}+w, \rho} \lambda(t) x(t) \\
& +c_{1} \frac{(t-a)^{q_{2}+w} e^{\frac{\rho-1}{\rho}(t-a)}}{\rho^{q_{2}+w} \Gamma\left(q_{2}+w+1\right)}+c_{2} \frac{(t-a)^{w} e^{\frac{\rho-1}{\rho}(t-a)}}{\rho^{w} \Gamma(w+1)} .
\end{aligned}
$$

Substituting $w=\left\{\mu_{i}, \beta_{j}, \gamma_{k}, \varphi_{l}\right\}, t=\left\{\sigma_{i}, \eta_{j}, \psi_{k}, \xi_{l}\right\}$ in (2.12), respectively, and applying the boundary conditions of problem (2.4), we have

$$
\begin{aligned}
\Omega_{1} c_{1}+\Omega_{2} c_{2}= & \sum_{j=1}^{n} \alpha_{j}\left[{ }_{a} I^{q_{1}+q_{2}+\beta_{j}, \rho} h\left(\eta_{j}\right)-{ }_{a} I^{q_{2}+\beta_{j}, \rho} \lambda\left(\eta_{j}\right) x\left(\eta_{j}\right)\right] \\
& -\sum_{i=1}^{m} \kappa_{i}\left[I^{I^{1}+q_{2}+\mu_{i}, \rho} h\left(\sigma_{i}\right)-{ }_{a} I^{q_{2}+\mu_{i}, \rho} \lambda\left(\sigma_{i}\right) x\left(\sigma_{i}\right)\right], \\
\Omega_{3} c_{1}+\Omega_{4} c_{2}= & \sum_{l=1}^{r} v_{l}\left[{ }_{a} I^{q_{1}+q_{2}+\varphi_{l}, \rho} h\left(\xi_{l}\right)-{ }_{a} I^{q_{2}+\varphi_{l}, \rho} \lambda\left(\xi_{l}\right) x\left(\xi_{l}\right)\right] \\
& -\sum_{k=1}^{p} \omega_{k}\left[{ }_{a} I^{q_{1}+q_{2}+\gamma_{k}, \rho} h\left(\psi_{k}\right)-{ }_{a} I^{q_{2}+\gamma_{k}, \rho} \lambda\left(\psi_{k}\right) x\left(\psi_{k}\right)\right] .
\end{aligned}
$$

Solving the above system for $c_{1}$ and $c_{2}$, we have

$$
\begin{aligned}
& c_{1}=\frac{1}{\Omega}\left[\Omega _ { 4 } \left(\sum_{j=1}^{n} \alpha_{j}\left[{ }_{a} I^{q_{1}+q_{2}+\beta_{j}, \rho} h\left(\eta_{j}\right)-{ }_{a} I^{q_{2}+\beta_{j}, \rho} \lambda\left(\eta_{j}\right) x\left(\eta_{j}\right)\right]\right.\right. \\
& \left.-\sum_{i=1}^{m} \kappa_{i}\left[I^{q_{1}+q_{2}+\mu_{i}, \rho} h\left(\sigma_{i}\right)-{ }_{a} I^{q_{2}+\mu_{i}, \rho} \lambda\left(\sigma_{i}\right) x\left(\sigma_{i}\right)\right]\right) \\
& -\Omega_{2}\left(\sum_{l=1}^{r} v_{l}\left[{ }_{a} I^{q_{1}+q_{2}+\varphi_{l}, \rho} h\left(\xi_{l}\right)-{ }_{a} I^{q_{2}+\varphi_{l}, \rho} \lambda\left(\xi_{l}\right) x\left(\xi_{l}\right)\right]\right. \\
& \left.\left.-\sum_{k=1}^{p} \omega_{k}\left[{ }_{a} I^{q_{1}+q_{2}+\gamma_{k}, \rho} h\left(\psi_{k}\right)-{ }_{a} I^{q_{2}+\gamma_{k}, \rho} \lambda\left(\psi_{k}\right) x\left(\psi_{k}\right)\right]\right)\right] \text {, } \\
& c_{2}=\frac{1}{\Omega}\left[\Omega _ { 1 } \left(\sum_{l=1}^{r} \nu_{l}\left[{ }_{a} I^{q_{1}+q_{2}+\varphi_{l}, \rho} h\left(\xi_{l}\right)-{ }_{a} I^{q_{2}+\varphi_{l}, \rho} \lambda\left(\xi_{l}\right) x\left(\xi_{l}\right)\right]\right.\right. \\
& \left.-\sum_{k=1}^{p} \omega_{k}\left[I^{q_{1}+q_{2}+\gamma_{k}, \rho} h\left(\psi_{k}\right)-{ }_{a} I^{q_{2}+\gamma_{k}, \rho} \lambda\left(\psi_{k}\right) x\left(\psi_{k}\right)\right]\right) \\
& -\Omega_{3}\left(\sum_{j=1}^{n} \alpha_{j}\left[I^{I^{q_{1}+q_{2}+\beta_{j}, \rho}} h\left(\eta_{j}\right)-{ }_{a} I^{q_{2}+\beta_{j}, \rho} \lambda\left(\eta_{j}\right) x\left(\eta_{j}\right)\right]\right.
\end{aligned}
$$




$$
\left.\left.-\sum_{i=1}^{m} \kappa_{i}\left[{ }_{a} I^{q_{1}+q_{2}+\mu_{i}, \rho} h\left(\sigma_{i}\right)-{ }_{a} I^{q_{2}+\mu_{i}, \rho} \lambda\left(\sigma_{i}\right) x\left(\sigma_{i}\right)\right]\right)\right],
$$

where $\Omega_{1}, \Omega_{2}, \Omega_{3}, \Omega_{4}$, and $\Omega$ are defined by (2.6)-(2.10), respectively. Substituting the values $c_{1}$ and $c_{2}$ into (2.11), we get the fractional integral equation (2.5).

Conversely, it is easily to shown by direct calculation that the solution $x(t)$ given by (2.5) satisfies problem (2.4) under the given boundary conditions. The proof is completed.

\section{Main results}

In this section, we establish the existence results of solutions for problem (1.1). Fixed point theorems are employed to prove the results.

Throughout this paper, the expression ${ }_{a} I^{b, \rho} f(s, x(s), x(\theta(s)),(\mathcal{K} x)(s))(c)$ means that

$$
\begin{aligned}
& { }_{a} I^{b, \rho} f(s, x(s), x(\theta(s)),(\mathcal{K} x)(s))(c) \\
& \quad=\frac{1}{\rho^{b} \Gamma(b)} \int_{a}^{c} e^{\frac{\rho-1}{\rho}(c-s)}(c-s)^{b-1} f(s, x(s), x(\theta(s)),(\mathcal{K} x)(s)) d s,
\end{aligned}
$$

where $b \in\left\{q_{2}, q_{1}+q_{2}, q_{2}+\mu_{i}, q_{2}+\beta_{j}, q_{2}+\gamma_{k}, q_{2}+\varphi_{l}, q_{1}+q_{2}+\mu_{i}, q_{1}+q_{2}+\beta_{j}, q_{1}+q_{2}+\gamma_{k}, q_{1}+\right.$ $\left.q_{2}+\varphi_{l}\right\}$ and $c \in\left\{t, \sigma_{i}, \eta_{j}, \psi_{k}, \xi_{l}\right\}$. For simplicity, we set

$$
F_{x}(t)=f(t, x(t), x(\theta(t)),(\mathcal{K} x)(t)) .
$$

In view of Lemma 2.8 , an operator $\mathcal{Q}: \mathbb{E} \rightarrow \mathbb{E}$ is defined by

$$
\begin{aligned}
& (\mathcal{Q} x)(t)={ }_{a} I^{q_{1}+q_{2}, \rho} F_{x}(s)(t)-{ }_{a} I^{q_{2, \rho} \rho} \lambda(s) x(s)(t) \\
& +\frac{e^{\frac{\rho-1}{\rho}(t-a)}}{\Omega}\left[\left(\frac{\Omega_{4}(t-a)^{q_{2}}}{\rho^{q_{2}} \Gamma\left(q_{2}+1\right)}-\Omega_{3}\right)\right. \\
& \times\left(\sum_{j=1}^{n} \alpha_{j}\left[a^{q^{q_{1}+q_{2}+\beta_{j}, \rho}} F_{x}(s)\left(\eta_{j}\right)-{ }_{a} I^{q_{2}+\beta_{j}, \rho} \lambda(s) x(s)\left(\eta_{j}\right)\right]\right. \\
& \left.-\sum_{i=1}^{m} \kappa_{i}\left[{ }_{a} I^{q_{1}+q_{2}+\mu_{i}, \rho} F_{x}(s)\left(\sigma_{i}\right)-{ }_{a} I^{q_{2}+\mu_{i}, \rho} \lambda(s) x(s)\left(\sigma_{i}\right)\right]\right) \\
& +\left(\Omega_{1}-\frac{\Omega_{2}(t-a)^{q_{2}}}{\rho^{q_{2}} \Gamma\left(q_{2}+1\right)}\right)\left(\sum_{l=1}^{r} \nu_{l}\left[a^{I^{q_{1}+q_{2}+\varphi_{l}, \rho}} F_{x}(s)\left(\xi_{l}\right)-{ }_{a} I^{q_{2}+\varphi_{l}, \rho} \lambda(s) x(s)\left(\xi_{l}\right)\right]\right. \\
& \left.\left.-\sum_{k=1}^{p} \omega_{k}\left[I^{q_{1}+q_{2}+\gamma_{k}, \rho} F_{x}(s)\left(\psi_{k}\right)-{ }_{a} I^{q_{2}+\gamma_{k}, \rho} \lambda(s) x(s)\left(\psi_{k}\right)\right]\right)\right] .
\end{aligned}
$$

Then problem (1.1) has solutions if and only if the operator $\mathcal{Q}$ has fixed points.

To proceed further, we introduce the following hypotheses:

$\left(H_{1}\right)$ Let $f:[a, T] \times \mathbb{R}^{3} \rightarrow \mathbb{R}$ be a continuous function.

$\left(H_{2}\right)$ Let $\lambda:[a, T] \rightarrow \mathbb{R}$ be a continuous function.

$\left(H_{3}\right)$ There exist positive constants $L_{1}, L_{2}$ such that

$$
\left|f\left(t, u_{1}, v_{1}, w_{1}\right)-f\left(t, u_{2}, v_{2}, w_{2}\right)\right| \leq L_{1}\left(\left|u_{1}-u_{2}\right|+\left|v_{1}-v_{2}\right|\right)+L_{2}\left|w_{1}-w_{2}\right|
$$

for any $u_{i}, v_{i}, w_{i} \in \mathbb{R}, i=1,2$, and $t \in[a, T]$. 
$\left(H_{4}\right)$ Let $\phi:[a, T]^{2} \times \mathbb{R} \rightarrow \mathbb{R}$ be a continuous function, and there exists a positive constant $\phi_{0}$ such that

$$
|\phi(t, s, u)-\phi(t, s, v)| \leq \phi_{0}|u-v|
$$

for each $(t, s) \in[a, T]^{2}$ and $u, v \in \mathbb{R}$.

$\left(H_{5}\right)|f(t, u, v, w)| \leq g(t), \forall(t, u, v, w) \in[a, T] \times \mathbb{R}^{3}$ and $g \in C\left([a, T], \mathbb{R}^{+}\right)$.

$\left(H_{6}\right)$ There exist nonnegative continuous functions $h_{1}, h_{2}, h_{3}, h_{4} \in \mathbb{E}$ such that

$$
|f(t, u, v, w)| \leq h_{1}(t)+h_{2}(t)|u|+h_{3}(t)|v|+h_{4}(t)|w|, \quad u, v, w \in \mathbb{R}, \quad t \in[a, T],
$$

with $h_{1}^{*}=\sup _{t \in[a, T]} h_{1}(t), \quad h_{2}^{*}=\sup _{t \in[a, T]} h_{2}(t), \quad h_{3}^{*}=\sup _{t \in[a, T]} h_{3}(t), \quad h_{4}^{*}=$ $\sup _{t \in[a, T]} h_{4}(t)$.

For the sake of computational convenience, we make use of the following constants:

$$
\begin{aligned}
& \Lambda_{1}=\frac{1}{|\Omega|}\left(\frac{\left|\Omega_{4}\right|(T-a)^{q_{2}}}{\rho^{q_{2}} \Gamma\left(q_{2}+1\right)}+\left|\Omega_{3}\right|\right) \\
& \Lambda_{2}=\frac{1}{|\Omega|}\left(\frac{\left|\Omega_{2}\right|(T-a)^{q_{2}}}{\rho^{q_{2}} \Gamma\left(q_{2}+1\right)}+\left|\Omega_{1}\right|\right) \text {, } \\
& \Lambda_{3}(u)=\frac{(T-a)^{u}}{\rho^{q_{1}+q_{2}} \Gamma(u+1)} \\
& +\Lambda_{1}\left(\sum_{j=1}^{n} \frac{\left|\alpha_{j}\right|\left(\eta_{j}-a\right)^{u+\beta_{j}}}{\rho^{q_{1}+q_{2}+\beta_{j}} \Gamma\left(u+\beta_{j}+1\right)}+\sum_{i=1}^{m} \frac{\left|\kappa_{i}\right|\left(\sigma_{i}-a\right)^{u+\mu_{i}}}{\rho^{q_{1}+q_{2}+\mu_{i}} \Gamma\left(u+\mu_{i}+1\right)}\right) \\
& +\Lambda_{2}\left(\sum_{l=1}^{r} \frac{\left|\nu_{l}\right|\left(\xi_{l}-a\right)^{u+\varphi_{l}}}{\rho^{q_{1}+q_{2}+\varphi_{l}} \Gamma\left(u+\varphi_{l}+1\right)}+\sum_{k=1}^{p} \frac{\left|\omega_{k}\right|\left(\psi_{k}-a\right)^{u+\gamma_{k}}}{\rho^{q_{1}+q_{2}+\gamma_{k}} \Gamma\left(u+\gamma_{k}+1\right)}\right) \text {, } \\
& \Lambda_{4}={ }_{a} I^{q_{2}, \rho}|\lambda(s)|(T)+\Lambda_{1}\left(\sum_{j=1}^{n}\left|\alpha_{j}\right|_{a} I^{q_{2}+\beta_{j}, \rho}|\lambda(s)|\left(\eta_{j}\right)+\sum_{i=1}^{m}\left|\kappa_{i}\right|_{a} I^{q_{2}+\mu_{i}, \rho}|\lambda(s)|\left(\sigma_{i}\right)\right) \\
& +\Lambda_{2}\left(\sum_{l=1}^{r}\left|v_{l}\right|_{a} I^{q_{2}+\varphi_{l}, \rho}|\lambda(s)|\left(\xi_{l}\right)+\sum_{k=1}^{p}\left|\omega_{k}\right|_{a} I^{q_{2}+\gamma_{k}, \rho}|\lambda(s)|\left(\psi_{k}\right)\right),
\end{aligned}
$$

where $u=\left\{q_{1}+q_{2}, q_{1}+q_{2}+1\right\}$.

\subsection{Existence and uniqueness result via Banach's contraction principle}

The existence and uniqueness result of a solution for problem (1.1) will be proved by using Banach's fixed point theorem.

Theorem 3.1 Suppose that hypotheses $\left(H_{1}\right),\left(H_{2}\right),\left(H_{3}\right)$, and $\left(H_{4}\right)$ are satisfied. If

$$
2 L_{1} \Lambda_{3}\left(q_{1}+q_{2}\right)+L_{2} \phi_{0} \Lambda_{3}\left(q_{1}+q_{2}+1\right)+\Lambda_{4}<1
$$

and $\Lambda_{1}, \Lambda_{2}, \Lambda_{3}(u), u=\left\{q_{1}+q_{2}, q_{1}+q_{2}+1\right\}$, and $\Lambda_{4}$ are given by (3.2), (3.3), (3.4), and (3.5), respectively, then problem (1.1) has a unique solution in the space $\mathbb{E}$. 
Proof Firstly, we transform problem (1.1) into a fixed point problem $x=\mathcal{Q} x$, where the operator $\mathcal{Q}$ is defined as in (3.1). Observe that the fixed points of the operator $\mathcal{Q}$ are solutions of problem (1.1). Applying the Banach contraction principle, we shall show that the operator $\mathcal{Q}$ has a fixed point which is the unique solution of problem (1.1).

Let $\sup _{t \in[a, T]}|f(t, 0,0,0)|:=M_{1}<\infty$. Next, we set $B_{R_{1}}:=\left\{x \in \mathbb{E}:\|x\|_{\mathbb{E}} \leq R_{1}\right\}$ with

$$
R_{1} \geq \frac{M_{1} \Lambda_{3}\left(q_{1}+q_{2}\right)}{1-\left[2 L_{1} \Lambda_{3}\left(q_{1}+q_{2}\right)-L_{2} \phi_{0} \Lambda_{3}\left(q_{1}+q_{2}+1\right)-\Lambda_{4}\right]},
$$

where $\Lambda_{3}(u), u=\left\{q_{1}+q_{2}, q_{1}+q_{2}+1\right\}$, and $\Lambda_{4}$ are given by (3.4) and (3.5), respectively. Note that $B_{R_{1}}$ is a bounded, closed, and convex subset of $\mathbb{E}$. The proof is divided into two steps as follows.

Step I. The operator $\mathcal{Q}$ defined by (3.1) satisfies the relation: $\mathcal{Q} B_{R_{1}} \subset B_{R_{1}}$.

For any $x \in B_{R_{1}}$, we have

$|(\mathcal{Q} x)(t)|$

$$
\begin{aligned}
& \leq{ }_{a} I^{q_{1}+q_{2}, \rho}\left|F_{x}(s)\right|(t)+{ }_{a} I^{q_{2}, \rho}|\lambda(s)||x(s)|(t)+\frac{e^{\frac{\rho-1}{\rho}(t-a)}}{|\Omega|}\left[\left(\frac{\left|\Omega_{4}\right|(t-a)^{q_{2}}}{\rho^{q_{2}} \Gamma\left(q_{2}+1\right)}+\left|\Omega_{3}\right|\right)\right. \\
& \times\left(\sum_{j=1}^{n}\left|\alpha_{j}\right|\left[{ }_{a} I^{q_{1}+q_{2}+\beta_{j}, \rho}\left|F_{x}(s)\right|\left(\eta_{j}\right)+{ }_{a} I^{q_{2}+\beta_{j}, \rho}|\lambda(s)||x(s)|\left(\eta_{j}\right)\right]\right. \\
& \left.+\sum_{i=1}^{m}\left|\kappa_{i}\right|\left[{ }_{a} I^{q_{1}+q_{2}+\mu_{i}, \rho}\left|F_{x}(s)\right|\left(\sigma_{i}\right)+{ }_{a} I^{q_{2}+\mu_{i}, \rho}|\lambda(s)||x(s)|\left(\sigma_{i}\right)\right]\right) \\
& +\left(\left|\Omega_{1}\right|+\frac{\left|\Omega_{2}\right|(t-a)^{q_{2}}}{\rho^{q_{2}} \Gamma\left(q_{2}+1\right)}\right)\left(\sum_{l=1}^{r}\left|v_{l}\right|\left[{ }_{a} I^{q_{1}+q_{2}+\varphi_{l}, \rho}\left|F_{x}(s)\right|\left(\xi_{l}\right)+{ }_{a} I^{q_{2}+\varphi_{l}, \rho}|\lambda(s)||x(s)|\left(\xi_{l}\right)\right]\right. \\
& \left.\left.+\sum_{k=1}^{p}\left|\omega_{k}\right|\left[a^{q_{1}+q_{2}+\gamma_{k}, \rho}\left|F_{x}(s)\right|\left(\psi_{k}\right)+{ }_{a} I^{q_{2}+\gamma_{k}, \rho}|\lambda(s)||x(s)|\left(\psi_{k}\right)\right]\right)\right] \\
& \leq{ }_{a} I^{q_{1}+q_{2}, \rho}\left(\left|F_{x}(s)-f(s, 0,0,0)\right|+|f(s, 0,0,0)|\right)(T)+{ }_{a} I^{q_{2}, \rho}|\lambda(s)||x(s)|(T)+\frac{e^{\frac{\rho-1}{\rho}(T-a)}}{|\Omega|} \\
& \times\left[( \frac { | \Omega _ { 4 } | ( T - a ) ^ { q _ { 2 } } } { \rho ^ { q _ { 2 } } \Gamma ( q _ { 2 } + 1 ) } + | \Omega _ { 3 } | ) \left(\sum _ { j = 1 } ^ { n } | \alpha _ { j } | \left[{ } _ { a } I ^ { q _ { 1 } + q _ { 2 } + \beta _ { j } , \rho } \left(\left|F_{x}(s)-f(s, 0,0,0)\right|\right.\right.\right.\right. \\
& \left.+|f(s, 0,0,0)|)\left(\eta_{j}\right)+{ }_{a} I^{q_{2}+\beta_{j}, \rho}|\lambda(s)||x(s)|\left(\eta_{j}\right)\right] \\
& +\sum_{i=1}^{m}\left|\kappa_{i}\right|\left[{ }_{a} I^{q_{1}+q_{2}+\mu_{i}, \rho}\left(\left|F_{x}(s)-f(s, 0,0,0)\right|+|f(s, 0,0,0)|\right)\left(\sigma_{i}\right)\right. \\
& \left.\left.+{ }_{a} I^{q_{2}+\mu_{i}, \rho}|\lambda(s)||x(s)|\left(\sigma_{i}\right)\right]\right)+\left(\left|\Omega_{1}\right|+\frac{\left|\Omega_{2}\right|(T-a)^{q_{2}}}{\rho^{q_{2}} \Gamma\left(q_{2}+1\right)}\right) \\
& \times\left(\sum _ { l = 1 } ^ { r } | \nu _ { l } | \left[{ }_{a} I^{q_{1}+q_{2}+\varphi_{l}, \rho}\left(\left|F_{x}(s)-f(s, 0,0,0)\right|+|f(s, 0,0,0)|\right)\left(\xi_{l}\right)\right.\right. \\
& \left.+{ }_{a} I^{q_{2}+\varphi_{l}, \rho}|\lambda(s)||x(s)|\left(\xi_{l}\right)\right]
\end{aligned}
$$




$$
\begin{aligned}
& +\sum_{k=1}^{p}\left|\omega_{k}\right|\left[{ }_{a} I^{q_{1}+q_{2}+\gamma_{k}, \rho}\left(\left|F_{x}(s)-f(s, 0,0,0)\right|+|f(s, 0,0,0)|\right)\left(\psi_{k}\right)\right. \\
& \left.\left.\left.+{ }_{a} I^{q_{2}+\gamma_{k}, \rho}|\lambda(s)||x(s)|\left(\psi_{k}\right)\right]\right)\right]
\end{aligned}
$$

By using the property $e^{\frac{\rho-1}{\rho}(u-s)} \leq 1$ for $a \leq s<u<t \leq T$ and $\left(H_{3}\right)-\left(H_{4}\right)$, we obtain

$$
\begin{aligned}
& |(\mathcal{Q} x)(t)| \\
& \leq{ }_{a} I^{q_{1}+q_{2}, \rho}\left(L_{1}(|x(s)|+|x(\theta(s))|)+L_{2}|(\mathcal{K} x)(s)|+M_{1}\right)(T)+{ }_{a} I^{q_{2}, \rho}|\lambda(s)||x(s)|(T) \\
& +\frac{e^{\frac{\rho-1}{\rho}(T-a)}}{|\Omega|}\left[\left(\frac{\left|\Omega_{4}\right|(T-a)^{q_{2}}}{\rho^{q_{2}} \Gamma\left(q_{2}+1\right)}+\left|\Omega_{3}\right|\right)\right. \\
& \times\left(\sum _ { j = 1 } ^ { n } | \alpha _ { j } | \left[{ }_{a} a^{q_{1}+q_{2}+\beta_{j}, \rho}\left(L_{1}(|x(s)|+|x(\theta(s))|)+L_{2}|(\mathcal{K} x)(s)|+M_{1}\right)\left(\eta_{j}\right)\right.\right. \\
& \left.+{ }_{a} I^{q_{2}+\beta_{j}, \rho}|\lambda(s)||x(s)|\left(\eta_{j}\right)\right]+\sum_{i=1}^{m}\left|\kappa_{i}\right|\left[a ^ { q ^ { q _ { 1 } + q _ { 2 } + \mu _ { i } , \rho } } \left(L_{1}(|x(s)|+|x(\theta(s))|)\right.\right. \\
& \left.\left.\left.+L_{2}|(\mathcal{K} x)(s)|+M_{1}\right)\left(\sigma_{i}\right)+{ }_{a} I^{q_{2}+\mu_{i}, \rho}|\lambda(s)||x(s)|\left(\sigma_{i}\right)\right]\right) \\
& +\left(\left|\Omega_{1}\right|+\frac{\left|\Omega_{2}\right|(T-a)^{q_{2}}}{\rho^{q_{2}} \Gamma\left(q_{2}+1\right)}\right)\left(\sum _ { l = 1 } ^ { r } | v _ { l } | \left[a ^ { I ^ { q _ { 1 } + q _ { 2 } + \varphi _ { l } , \rho } } \left(L_{1}(|x(s)|+|x(\theta(s))|)\right.\right.\right. \\
& \left.\left.+L_{2}|(\mathcal{K} x)(s)|+M_{1}\right)\left(\xi_{l}\right)+{ }_{a} I^{q_{2}+\varphi_{l}, \rho}|\lambda(s)||x(s)|\left(\xi_{l}\right)\right] \\
& +\sum_{k=1}^{p}\left|\omega_{k}\right|\left[I ^ { q _ { 1 } + q _ { 2 } + \gamma _ { k } , \rho } \left(L_{1}(|x(s)|+|x(\theta(s))|)\right.\right. \\
& \left.\left.\left.\left.+L_{2}|(\mathcal{K} x)(s)|+M_{1}\right)\left(\psi_{k}\right)+{ }_{a} I^{q_{2}+\gamma_{k}, \rho}|\lambda(s)||x(s)|\left(\psi_{k}\right)\right]\right)\right] \\
& \leq \frac{1}{\rho^{q_{1}+q_{2}} \Gamma\left(q_{1}+q_{2}\right)} \int_{a}^{T}(T-s)^{q_{1}+q_{2}-1}\left(2 L_{1} R_{1}+L_{2} \phi_{0}(s-a) R_{1}+M_{1}\right) d s \\
& +R_{1 a} I^{q_{2}, \rho}|\lambda(s)|(T)+\frac{1}{|\Omega|}\left[\left(\frac{\left|\Omega_{4}\right|(T-a)^{q_{2}}}{\rho^{q_{2}} \Gamma\left(q_{2}+1\right)}+\left|\Omega_{3}\right|\right)\right. \\
& \times\left(\sum _ { j = 1 } ^ { n } | \alpha _ { j } | \left[\frac{1}{\rho^{q_{1}+q_{2}+\beta_{j}} \Gamma\left(q_{1}+q_{2}+\beta_{j}\right)} \int_{a}^{\eta_{j}}\left(\eta_{j}-s\right)^{q_{1}+q_{2}+\beta_{j}-1}\right.\right. \\
& \left.\times\left(2 L_{1} R_{1}+L_{2} \phi_{0}(s-a) R_{1}+M_{1}\right) d s+R_{1 a} I^{q_{2}+\beta_{j}, \rho}|\lambda(s)|\left(\eta_{j}\right)\right] \\
& +\sum_{i=1}^{m}\left|\kappa_{i}\right|\left[\frac{1}{\rho^{q_{1}+q_{2}+\mu_{i}} \Gamma\left(q_{1}+q_{2}+\mu_{i}\right)}\right. \\
& \left.\left.\times \int_{a}^{\sigma_{i}}\left(\sigma_{i}-s\right)^{q_{1}+q_{2}+\mu_{i}-1}\left(2 L_{1} R_{1}+L_{2} \phi_{0}(s-a) R_{1}+M_{1}\right) d s+R_{1 a} I^{q_{2}+\mu_{i}, \rho}|\lambda(s)|\left(\sigma_{i}\right)\right]\right)
\end{aligned}
$$




$$
\begin{aligned}
& +\left(\left|\Omega_{1}\right|+\frac{\left|\Omega_{2}\right|(T-a)^{q_{2}}}{\rho^{q_{2}} \Gamma\left(q_{2}+1\right)}\right)\left(\sum _ { l = 1 } ^ { r } | v _ { l } | \left[\frac{1}{\rho^{q_{1}+q_{2}+\varphi_{l}} \Gamma\left(q_{1}+q_{2}+\varphi_{l}\right)} \int_{a}^{\xi_{l}}\left(\xi_{l}-s\right)^{q_{1}+q_{2}+\varphi_{l}-1}\right.\right. \\
& \left.\times\left(2 L_{1} R_{1}+L_{2} \phi_{0}(s-a) R_{1}+M_{1}\right) d s+R_{1 a} I^{q_{2}+\varphi_{l}, \rho}|\lambda(s)|\left(\xi_{l}\right)\right] \\
& +\sum_{k=1}^{p}\left|\omega_{k}\right|\left[\frac{1}{\rho^{q_{1}+q_{2}+\gamma_{k}} \Gamma\left(q_{1}+q_{2}+\gamma_{k}\right)}\right. \\
& \times \int_{a}^{\psi_{k}}\left(\psi_{k}-s\right)^{q_{1}+q_{2}+\gamma_{k}-1}\left(2 L_{1} R_{1}+L_{2} \phi_{0}(s-a) R_{1}+M_{1}\right) d s \\
& \left.\left.\left.+R_{1 a} I^{q_{2}+\gamma_{k}, \rho}|\lambda(s)|\left(\psi_{k}\right)\right]\right)\right] \\
& \leq\left(2 L_{1} R_{1}+M_{1}\right)\left[\frac{(T-a)^{q_{1}+q_{2}}}{\rho^{q_{1}+q_{2}} \Gamma\left(q_{1}+q_{2}+1\right)}+\Lambda_{1}\left(\sum_{j=1}^{n} \frac{\left|\alpha_{j}\right|\left(\eta_{j}-a\right)^{q_{1}+q_{2}+\beta_{j}}}{\rho^{q_{1}+q_{2}+\beta_{j}} \Gamma\left(q_{1}+q_{2}+\beta_{j}+1\right)}\right.\right. \\
& \left.+\sum_{i=1}^{m} \frac{\left|\kappa_{i}\right|\left(\sigma_{i}-a\right)^{q_{1}+q_{2}+\mu_{i}}}{\rho^{q_{1}+q_{2}+\mu_{i}} \Gamma\left(q_{1}+q_{2}+\mu_{i}+1\right)}\right)+\Lambda_{2}\left(\sum_{l=1}^{r} \frac{\left|\nu_{l}\right|\left(\xi_{l}-a\right)^{q_{1}+q_{2}+\varphi_{l}}}{\rho^{q_{1}+q_{2}+\varphi_{l}} \Gamma\left(q_{1}+q_{2}+\varphi_{l}+1\right)}\right. \\
& \left.\left.+\sum_{k=1}^{p} \frac{\left|\omega_{k}\right|\left(\psi_{k}-a\right)^{q_{1}+q_{2}+\gamma_{k}}}{\rho^{q_{1}+q_{2}+\gamma_{k}} \Gamma\left(q_{1}+q_{2}+\gamma_{k}+1\right)}\right)\right]+L_{2} \phi_{0} R_{1}\left[\frac{(T-a)^{q_{1}+q_{2}+1}}{\rho^{q_{1}+q_{2}} \Gamma\left(q_{1}+q_{2}+2\right)}\right. \\
& +\Lambda_{1}\left(\sum_{j=1}^{n} \frac{\left|\alpha_{j}\right|\left(\eta_{j}-a\right)^{q_{1}+q_{2}+\beta_{j}+1}}{\rho^{q_{1}+q_{2}+\beta_{j}} \Gamma\left(q_{1}+q_{2}+\beta_{j}+2\right)}+\sum_{i=1}^{m} \frac{\left|\kappa_{i}\right|\left(\sigma_{i}-a\right)^{q_{1}+q_{2}+\mu_{i}+1}}{\rho^{q_{1}+q_{2}+\mu_{i}} \Gamma\left(q_{1}+q_{2}+\mu_{i}+2\right)}\right) \\
& \left.+\Lambda_{2}\left(\sum_{l=1}^{r} \frac{\left|v_{l}\right|\left(\xi_{l}-a\right)^{q_{1}+q_{2}+\varphi_{l}+1}}{\rho^{q_{1}+q_{2}+\varphi_{l}} \Gamma\left(q_{1}+q_{2}+\varphi_{l}+2\right)}+\sum_{k=1}^{p} \frac{\left|\omega_{k}\right|\left(\psi_{k}-a\right)^{q_{1}+q_{2}+\gamma_{k}+1}}{\rho^{q_{1}+q_{2}+\gamma_{k}} \Gamma\left(q_{1}+q_{2}+\gamma_{k}+2\right)}\right)\right] \\
& +R_{1}\left[{ }_{a} I^{q_{2}, \rho}|\lambda(s)|(T)+\Lambda_{1}\left(\sum_{j=1}^{n}\left|\alpha_{j}\right|_{a} I^{q_{2}+\beta_{j}, \rho}|\lambda(s)|\left(\eta_{j}\right)+\sum_{i=1}^{m}\left|\kappa_{i}\right|_{a} I^{q_{2}+\mu_{i}, \rho}|\lambda(s)|\left(\sigma_{i}\right)\right)\right. \\
& \left.+\Lambda_{2}\left(\sum_{l=1}^{r}\left|\nu_{l}\right|_{a} I^{q_{2}+\varphi_{l}, \rho}|\lambda(s)|\left(\xi_{l}\right)+\sum_{k=1}^{p}\left|\omega_{k}\right|_{a} I^{q_{2}+\gamma_{k}, \rho}|\lambda(s)|\left(\psi_{k}\right)\right)\right] \\
& \leq\left(2 L_{1} R_{1}+M_{1}\right) \Lambda_{3}(u)+L_{2} \phi_{0} R_{1} \Lambda_{3}(u+1)+R_{1} \Lambda_{4} \leq R_{1},
\end{aligned}
$$

which implies that $\|\mathcal{Q} x\|_{\mathbb{E}} \leq R_{1}$. Therefore, $\mathcal{Q}$ maps bounded subsets of $B_{R_{1}}$ into bounded subsets of $B_{R_{1}}$, that is, $\mathcal{Q} B_{R_{1}} \subset B_{R_{1}}$.

Step II. To show that an operator $\mathcal{Q}: \mathbb{E} \rightarrow \mathbb{E}$ is contraction.

For any $x y \in \mathbb{E}$ and for each $t \in[a, T]$, we have

$$
\begin{aligned}
& |(\mathcal{Q} x)(t)-(\mathcal{Q} y)(t)| \\
& \leq a^{q_{1}+q_{2}, \rho}\left|F_{x}(s)-F_{y}(s)\right|(T)+{ }_{a} I^{q_{2}, \rho}|\lambda(s)||x(s)-y(s)|(T) \\
& \quad+\frac{e^{\frac{\rho-1}{\rho}(T-a)}}{|\Omega|}\left[\left(\frac{\left|\Omega_{4}\right|(T-a)^{q_{2}}}{\rho^{q_{2}} \Gamma\left(q_{2}+1\right)}+\left|\Omega_{3}\right|\right)\right. \\
& \quad \times\left(\sum_{j=1}^{n}\left|\alpha_{j}\right|\left[{ }_{a} I^{q_{1}+q_{2}+\beta_{j}, \rho}\left|F_{x}(s)-F_{y}(s)\right|\left(\eta_{j}\right)+{ }_{a} I^{q_{2}+\beta_{j}, \rho}|\lambda(s)||x(s)-y(s)|\left(\eta_{j}\right)\right]\right.
\end{aligned}
$$




$$
\begin{aligned}
& \left.+\sum_{i=1}^{m}\left|\kappa_{i}\right|\left[{ }_{a} I^{q_{1}+q_{2}+\mu_{i}, \rho}\left|F_{x}(s)-F_{y}(s)\right|\left(\sigma_{i}\right)+{ }_{a} I^{q_{2}+\mu_{i}, \rho}|\lambda(s)||x(s)-y(s)|\left(\sigma_{i}\right)\right]\right) \\
& +\left(\frac{\left|\Omega_{2}\right|(T-a)^{q_{2}}}{\rho^{q_{2}} \Gamma\left(q_{2}+1\right)}+\left|\Omega_{1}\right|\right)\left(\sum _ { l = 1 } ^ { r } | v _ { l } | \left[a^{q_{1}+q_{2}+\varphi_{l}, \rho}\left|F_{x}(s)-F_{y}(s)\right|\left(\xi_{l}\right)\right.\right. \\
& \left.+{ }_{a} I^{q_{2}+\varphi_{l}, \rho}|\lambda(s)||x(s)-y(s)|\left(\xi_{l}\right)\right]+\sum_{k=1}^{p}\left|\omega_{k}\right|\left[I^{q_{1}+q_{2}+\gamma_{k}, \rho}\left|F_{x}(s)-F_{y}(s)\right|\left(\psi_{k}\right)\right. \\
& \left.\left.\left.+{ }_{a} I^{q_{2}+\gamma_{k}, \rho}|\lambda(s)||x(s)-y(s)|\left(\psi_{k}\right)\right]\right)\right] \\
& \leq{ }_{a} I^{q_{1}+q_{2}, \rho}\left(2 L_{1}+L_{2} \phi_{0}(s-a)\right)(T)\|x-y\|_{\mathbb{E}}+{ }_{a} I^{q_{2}, \rho}|\lambda(s)|(T)\|x-y\|_{\mathbb{E}} \\
& +\frac{e^{\frac{\rho-1}{\rho}(T-a)}}{|\Omega|}\left[\left(\frac{\left|\Omega_{4}\right|(T-a)^{q_{2}}}{\rho^{q_{2}} \Gamma\left(q_{2}+1\right)}+\left|\Omega_{3}\right|\right)\right. \\
& \times\left(\sum _ { j = 1 } ^ { n } | \alpha _ { j } | \left[a_{a} I^{q_{1}+q_{2}+\beta_{j}, \rho}\left(2 L_{1}+L_{2} \phi_{0}(s-a)\right)\left(\eta_{j}\right)\|x-y\|_{\mathbb{E}}\right.\right. \\
& \left.+{ }_{a} I^{q_{2}+\beta_{j}, \rho}|\lambda(s)|\left(\eta_{j}\right)\|x-y\|_{\mathbb{E}}\right] \\
& +\sum_{i=1}^{m}\left|\kappa_{i}\right|\left[{ }_{a} I^{q_{1}+q_{2}+\mu_{i}, \rho}\left(2 L_{1}+L_{2} \phi_{0}(s-a)\right)\left(\sigma_{i}\right)\|x-y\|_{\mathbb{E}}\right. \\
& \left.\left.+{ }_{a} I^{q_{2}+\mu_{i}, \rho}|\lambda(s)|\left(\sigma_{i}\right)\|x-y\|_{\mathbb{E}}\right]\right)+\left(\frac{\left|\Omega_{2}\right|(T-a)^{q_{2}}}{\rho^{q_{2}} \Gamma\left(q_{2}+1\right)}+\left|\Omega_{1}\right|\right) \\
& \times\left(\sum _ { l = 1 } ^ { r } | v _ { l } | \left[{ }_{a} I^{q_{1}+q_{2}+\varphi_{l}, \rho}\left(2 L_{1}+L_{2} \phi_{0}(s-a)\right)\left(\xi_{l}\right)\|x-y\|_{\mathbb{E}}\right.\right. \\
& \left.+{ }_{a} I^{q_{2}+\varphi_{l}, \rho}|\lambda(s)|\left(\xi_{l}\right)\|x-y\|_{\mathbb{E}}\right] \\
& +\sum_{k=1}^{p}\left|\omega_{k}\right|\left[a^{q_{1}+q_{2}+\gamma_{k}, \rho}\left(2 L_{1}+L_{2} \phi_{0}(s-a)\right)\left(\psi_{k}\right)\|x-y\|_{\mathbb{E}}\right. \\
& \left.\left.\left.+{ }_{a} I^{q_{2}+\gamma_{k}, \rho}|\lambda(s)|\left(\psi_{k}\right)\|x-y\|_{\mathbb{E}}\right]\right)\right] \\
& \leq\left[2 L _ { 1 } \left[\frac{(T-a)^{q_{1}+q_{2}}}{\rho^{q_{1}+q_{2}} \Gamma\left(q_{1}+q_{2}+1\right)}+\Lambda_{1}\left(\sum_{j=1}^{n} \frac{\left|\alpha_{j}\right|\left(\eta_{j}-a\right)^{q_{1}+q_{2}+\beta_{j}}}{\rho^{q_{1}+q_{2}+\beta_{j}} \Gamma\left(q_{1}+q_{2}+\beta_{j}+1\right)}\right.\right.\right. \\
& \left.+\sum_{i=1}^{m} \frac{\left|\kappa_{i}\right|\left(\sigma_{i}-a\right)^{q_{1}+q_{2}+\mu_{i}}}{\rho^{q_{1}+q_{2}+\mu_{i}} \Gamma\left(q_{1}+q_{2}+\mu_{i}+1\right)}\right)+\Lambda_{2}\left(\sum_{l=1}^{r} \frac{\left|v_{l}\right|\left(\xi_{l}-a\right)^{q_{1}+q_{2}+\varphi_{l}}}{\rho^{q_{1}+q_{2}+\varphi_{l}} \Gamma\left(q_{1}+q_{2}+\varphi_{l}+1\right)}\right. \\
& \left.\left.+\sum_{k=1}^{p} \frac{\left|\omega_{k}\right|\left(\psi_{k}-a\right)^{q_{1}+q_{2}+\gamma_{k}}}{\rho^{q_{1}+q_{2}+\gamma_{k}} \Gamma\left(q_{1}+q_{2}+\gamma_{k}+1\right)}\right)\right]+L_{2} \phi_{0}\left[\frac{(T-a)^{q_{1}+q_{2}+1}}{\rho^{q_{1}+q_{2}} \Gamma\left(q_{1}+q_{2}+2\right)}\right. \\
& +\Lambda_{1}\left(\sum_{j=1}^{n} \frac{\left|\alpha_{j}\right|\left(\eta_{j}-a\right)^{q_{1}+q_{2}+\beta_{j}+1}}{\rho^{q_{1}+q_{2}+\beta_{j}} \Gamma\left(q_{1}+q_{2}+\beta_{j}+2\right)}+\sum_{i=1}^{m} \frac{\left|\kappa_{i}\right|\left(\sigma_{i}-a\right)^{q_{1}+q_{2}+\mu_{i}+1}}{\rho^{q_{1}+q_{2}+\mu_{i}} \Gamma\left(q_{1}+q_{2}+\mu_{i}+2\right)}\right) \\
& \left.+\Lambda_{2}\left(\sum_{l=1}^{r} \frac{\left|\nu_{l}\right|\left(\xi_{l}-a\right)^{q_{1}+q_{2}+\varphi_{l}+1}}{\rho^{q_{1}+q_{2}+\varphi_{l}} \Gamma\left(q_{1}+q_{2}+\varphi_{l}+2\right)}+\sum_{k=1}^{p} \frac{\left|\omega_{k}\right|\left(\psi_{k}-a\right)^{q_{1}+q_{2}+\gamma_{k}+1}}{\rho^{q_{1}+q_{2}+\gamma_{k}} \Gamma\left(q_{1}+q_{2}+\gamma_{k}+2\right)}\right)\right]
\end{aligned}
$$




$$
\begin{aligned}
& +{ }_{a} I^{q_{2}, \rho}|\lambda(s)|(T)+\Lambda_{1}\left(\sum_{j=1}^{n}\left|\alpha_{j}\right|_{a} I^{q_{2}+\beta_{j}, \rho}|\lambda(s)|\left(\eta_{j}\right)+\sum_{i=1}^{m}\left|\kappa_{i}\right|_{a} I^{q_{2}+\mu_{i}, \rho}|\lambda(s)|\left(\sigma_{i}\right)\right) \\
& \left.+\Lambda_{2}\left(\sum_{l=1}^{r}\left|v_{l}\right|_{a} I^{q_{2}+\varphi_{l}, \rho}|\lambda(s)|\left(\xi_{l}\right)+\sum_{k=1}^{p}\left|\omega_{k}\right|_{a} I^{q_{2}+\gamma_{k}, \rho}|\lambda(s)|\left(\psi_{k}\right)\right)\right]\|x-y\|_{\mathbb{E}} \\
& \leq\left[2 L_{1} \Lambda_{3}\left(q_{1}+q_{2}\right)+L_{2} \phi_{0} \Lambda_{3}\left(q_{1}+q_{2}+1\right)+\Lambda_{4}\right]\|x-y\|_{\mathbb{E}},
\end{aligned}
$$

which implies that $\|\mathcal{Q} x-\mathcal{Q} y\|_{\mathbb{E}} \leq\left[2 L_{1} \Lambda_{3}\left(q_{1}+q_{2}\right)+L_{2} \phi_{0} \Lambda_{3}\left(q_{1}+q_{2}+1\right)+\Lambda_{4}\right]\|x-y\|_{\mathbb{E}}$. As $2 L_{1} \Lambda_{3}\left(q_{1}+q_{2}\right)+L_{2} \phi_{0} \Lambda_{3}\left(q_{1}+q_{2}+1\right)+\Lambda_{4}<1$, hence, by the Banach contraction principle (Lemma 2.5), the operator $\mathcal{Q}$ is a contraction, therefore, it has a unique fixed point that is the unique solution of problem (1.1) in $\mathbb{E}$. The proof is completed.

\subsection{Existence result via Krasnoselskii's fixed point theorem}

By using Krasnoselskii's fixed point theorem, the existence theorem will be obtained.

Theorem 3.2 Assume that $\left(H_{1}\right),\left(H_{2}\right),\left(H_{4}\right)$, and $\left(H_{5}\right)$ hold. Then problem (1.1) has at least one solution on $[a, T]$ provided $\Lambda_{4}<1$, where $\Lambda_{4}$ is defined by (3.5).

Proof Let $\sup _{t \in[a, T]}|g(t)|=\|g\|_{\mathbb{E}}$. By choosing a suitable $B_{\bar{R}_{2}}=\left\{x \in \mathbb{E}:\|x\|_{\mathbb{E}} \leq \bar{R}_{2}\right\}$, where

$$
\bar{R}_{2} \geq \frac{\Lambda_{3}\left(q_{1}+q_{2}\right)\|g\|_{\mathbb{E}}}{1-\Lambda_{4}}
$$

and $\Lambda_{3}\left(q_{1}+q_{2}\right)$ and $\Lambda_{4}$ are defined by (3.4) and (3.5), respectively. We define the operators $\mathcal{Q}_{1}$ and $\mathcal{Q}_{2}$ on $B_{\bar{R}_{2}}$ by

$$
\begin{aligned}
\left(\mathcal{Q}_{1} x\right)(t)= & { }_{a} I^{q_{1}+q_{2}, \rho} F_{x}(s)(t)+\frac{e^{\frac{\rho-1}{\rho}(t-a)}}{\Omega}\left[\left(\frac{\Omega_{4}(t-a)^{q_{2}}}{\rho^{q_{2}} \Gamma\left(q_{2}+1\right)}-\Omega_{3}\right)\right. \\
& \times\left(\sum_{j=1}^{n} \alpha_{j a} I^{q_{1}+q_{2}+\beta_{j}, \rho} F_{x}(s)\left(\eta_{j}\right)-\sum_{i=1}^{m} \kappa_{i a} I^{q_{1}+q_{2}+\mu_{i}, \rho} F_{x}(s)\left(\sigma_{i}\right)\right) \\
& +\left(\Omega_{1}-\frac{\Omega_{2}(t-a)^{q_{2}}}{\rho^{q_{2}} \Gamma\left(q_{2}+1\right)}\right) \\
& \left.\times\left(\sum_{l=1}^{r} v_{l a} I^{q_{1}+q_{2}+\varphi_{l}, \rho} F_{x}(s)\left(\xi_{l}\right)-\sum_{k=1}^{p} \omega_{k a} I^{I_{1}+q_{2}+\gamma_{k}, \rho} F_{x}(s)\left(\psi_{k}\right)\right)\right], \quad t \in[a, T], \\
\left(\mathcal{Q}_{2} x\right)(t)= & \frac{e^{\frac{\rho-1}{\rho}(t-a)}}{\Omega}\left[( \frac { \Omega _ { 4 } ( t - a ) ^ { q _ { 2 } } } { \rho ^ { q _ { 2 } } \Gamma ( q _ { 2 } + 1 ) } - \Omega _ { 3 } ) \left(\sum_{i=1}^{m} \kappa_{i a} I^{q_{2}+\mu_{i}, \rho} \lambda(s) x(s)\left(\sigma_{i}\right)\right.\right. \\
& \left.-\sum_{j=1}^{n} \alpha_{j a} I^{q_{2}+\beta_{j}, \rho} \lambda(s) x(s)\left(\eta_{j}\right)\right) \\
& +\left(\Omega_{1}-\frac{\Omega_{2}(t-a)^{q_{2}}}{\rho^{q_{2}} \Gamma\left(q_{2}+1\right)}\right)\left(\sum_{k=1}^{p} \omega_{k a} I^{q_{2}+\gamma_{k}, \rho} \lambda(s) x(s)\left(\psi_{k}\right)\right. \\
& \left.\left.-\sum_{l=1}^{r} v_{l a} I^{q_{2}+\varphi_{l}, \rho} \lambda(s) x(s)\left(\xi_{l}\right)\right)\right]-{ }_{a} I^{q_{2}, \rho} \lambda(s) x(s)(t), \quad t \in[a, T] .
\end{aligned}
$$


Note that $\mathcal{Q}=\mathcal{Q}_{1}+\mathcal{Q}_{2}$. For any $x, y \in B_{\bar{R}_{2}}$, we have

$$
\begin{aligned}
& \left\|\mathcal{Q}_{1} x+\mathcal{Q}_{2} y\right\|_{\mathbb{E}} \\
& \leq \sup _{t \in[a, T]}\left\{{ }_{a} I^{q_{1}+q_{2}, \rho}\left|F_{x}(s)\right|(t)+{ }_{a} I^{q_{2}, \rho}|\lambda(s)||y(s)|(t)\right. \\
& +\frac{e^{\frac{\rho-1}{\rho}(t-a)}}{|\Omega|}\left[\left(\frac{\left|\Omega_{4}\right|(t-a)^{q_{2}}}{\rho^{q_{2}} \Gamma\left(q_{2}+1\right)}+\left|\Omega_{3}\right|\right)\right. \\
& \times\left(\sum_{j=1}^{n}\left|\alpha_{j}\right|\left[{ }_{a} I^{q_{1}+q_{2}+\beta_{j}, \rho}\left|F_{x}(s)\right|\left(\eta_{j}\right)+{ }_{a} I^{q_{2}+\beta_{j}, \rho}|\lambda(s)||y(s)|\left(\eta_{j}\right)\right]\right. \\
& \left.+\sum_{i=1}^{m}\left|\kappa_{i}\right|\left[{ }_{a} I^{q_{1}+q_{2}+\mu_{i}, \rho}\left|F_{x}(s)\right|\left(\sigma_{i}\right)+{ }_{a} I^{q_{2}+\mu_{i}, \rho}|\lambda(s)||y(s)|\left(\sigma_{i}\right)\right]\right) \\
& +\left(\left|\Omega_{1}\right|+\frac{\left|\Omega_{2}\right|(t-a)^{q_{2}}}{\rho^{q_{2}} \Gamma\left(q_{2}+1\right)}\right)\left(\sum _ { l = 1 } ^ { r } | \nu _ { l } | \left[{ }_{a} I^{q_{1}+q_{2}+\varphi_{l}, \rho}\left|F_{x}(s)\right|\left(\xi_{l}\right)\right.\right. \\
& \left.+{ }_{a} I^{q_{2}+\varphi_{l}, \rho}|\lambda(s)||y(s)|\left(\xi_{l}\right)\right] \\
& \left.\left.\left.+\sum_{k=1}^{p}\left|\omega_{k}\right|\left[{ }_{a} I^{q_{1}+q_{2}+\gamma_{k}, \rho}\left|F_{x}(s)\right|\left(\psi_{k}\right)+{ }_{a} I^{q_{2}+\gamma_{k}, \rho}|\lambda(s)||y(s)|\left(\psi_{k}\right)\right]\right)\right]\right\} \\
& \leq\|g\|_{\mathbb{E}}\left[\frac{(T-a)^{q_{1}+q_{2}}}{\rho^{q_{1}+q_{2}} \Gamma\left(q_{1}+q_{2}+1\right)}+\Lambda_{1}\left(\sum_{j=1}^{n} \frac{\left|\alpha_{j}\right|\left(\eta_{j}-a\right)^{q_{1}+q_{2}+\beta_{j}}}{\rho^{q_{1}+q_{2}+\beta_{j}} \Gamma\left(q_{1}+q_{2}+\beta_{j}+1\right)}\right.\right. \\
& \left.+\sum_{i=1}^{m} \frac{\left.\left|\kappa_{i}\right|\left(\sigma_{i}-a\right)^{q_{1}+q_{2}+\mu_{i}}\right)}{\rho^{q_{1}+q_{2}+\mu_{i}} \Gamma\left(q_{1}+q_{2}+\mu_{i}+1\right)}\right)+\Lambda_{2}\left(\sum_{l=1}^{r} \frac{\left|\nu_{l}\right|\left(\xi_{l}-a\right)^{q_{1}+q_{2}+\varphi_{l}}}{\rho^{q_{1}+q_{2}+\varphi_{l}} \Gamma\left(q_{1}+q_{2}+\varphi_{l}+1\right)}\right. \\
& \left.\left.+\sum_{k=1}^{p} \frac{\left|\omega_{k}\right|\left(\psi_{k}-a\right)^{q_{1}+q_{2}+\gamma_{k}}}{\rho^{q_{1}+q_{2}+\gamma_{k}} \Gamma\left(q_{1}+q_{2}+\gamma_{k}+1\right)}\right)\right] \\
& +\bar{R}_{2}\left[{ }_{a} I^{q_{2}, \rho}|\lambda(s)|(T)+\Lambda_{1}\left(\sum_{j=1}^{n}\left|\alpha_{j}\right|_{a} I^{q_{2}+\beta_{j}, \rho}|\lambda(s)|\left(\eta_{j}\right)+\sum_{i=1}^{m}\left|\kappa_{i}\right|_{a} I^{q_{2}+\mu_{i}, \rho}|\lambda(s)|\left(\sigma_{i}\right)\right)\right. \\
& \left.+\Lambda_{2}\left(\sum_{l=1}^{r}\left|\nu_{l}\right|_{a} I^{q_{2}+\varphi_{l}, \rho}|\lambda(s)|\left(\xi_{l}\right)+\sum_{k=1}^{p}\left|\omega_{k}\right|_{a} I^{q_{2}+\gamma_{k}, \rho}|\lambda(s)|\left(\psi_{k}\right)\right)\right] \\
& =\Lambda_{3}\left(q_{1}+q_{2}\right)\|g\|_{\mathbb{E}}+\Lambda_{4} \bar{R}_{2} \leq \bar{R}_{2},
\end{aligned}
$$

which implies that $\left\|\mathcal{Q}_{1} x+\mathcal{Q}_{2} y\right\| \leq B_{\bar{R}_{2}}$. It follows that $\mathcal{Q}_{1} x+\mathcal{Q}_{2} y \in B_{\bar{R}_{2}}$, which satisfies assumption (i) of Lemma 2.6.

To show that assumption (ii) of Lemma 2.6 is satisfied, let $\left\{x_{n}\right\}$ be a sequence such that $x_{n} \rightarrow x$ in $\mathbb{E}$. Then, for each $t \in[a, T]$, we take

$$
\begin{aligned}
& \left|\left(\mathcal{Q}_{1} x_{n}\right)(t)-\left(\mathcal{Q}_{1} x\right)(t)\right| \\
& \quad \leq{ }_{a} I^{q_{1}+q_{2}, \rho}\left|F_{x_{n}}(s)-F_{x}(s)\right|(T)+\frac{1}{|\Omega|}\left[\left(\frac{\left|\Omega_{4}\right|(T-a)^{q_{2}}}{\rho^{q_{2}} \Gamma\left(q_{2}+1\right)}+\left|\Omega_{3}\right|\right)\right.
\end{aligned}
$$




$$
\begin{aligned}
& \times\left(\sum_{j=1}^{n}\left|\alpha_{j}\right|_{a} I^{q_{1}+q_{2}+\beta_{j}, \rho}\left|F_{x_{n}}(s)-F_{x}(s)\right|\left(\eta_{j}\right)+\sum_{i=1}^{m}\left|\kappa_{i}\right|_{a} I^{q_{1}+q_{2}+\mu_{i}, \rho}\left|F_{x_{n}}(s)-F_{x}(s)\right|\left(\sigma_{i}\right)\right) \\
& +\left(\left|\Omega_{1}\right|+\frac{\left|\Omega_{2}\right|(T-a)^{q_{2}}}{\rho^{q_{2}} \Gamma\left(q_{2}+1\right)}\right)\left(\sum_{l=1}^{r}\left|v_{l}\right|_{a} I^{q_{1}+q_{2}+\varphi_{l}, \rho}\left|F_{x_{n}}(s)-F_{x}(s)\right|\left(\xi_{l}\right)\right. \\
& \left.\left.+\sum_{k=1}^{p}\left|\omega_{k}\right|_{a} I^{q_{1}+q_{2}+\gamma_{k}, \rho}\left|F_{x_{n}}(s)-F_{x}(s)\right|\left(\psi_{k}\right)\right)\right] \\
& \leq\left\{\frac{(T-a)^{q_{1}+q_{2}}}{\rho^{q_{1}+q_{2}} \Gamma\left(q_{1}+q_{2}+1\right)}\right. \\
& +\Lambda_{1}\left(\sum_{j=1}^{n} \frac{\left|\alpha_{j}\right|\left(\eta_{j}-a\right)^{q_{1}+q_{2}+\beta_{j}}}{\rho^{q_{1}+q_{2}+\beta_{j}} \Gamma\left(q_{1}+q_{2}+\beta_{j}+1\right)}+\sum_{i=1}^{m} \frac{\left|\kappa_{i}\right|\left(\sigma_{i}-a\right)^{q_{1}+q_{2}+\mu_{i}}}{\rho^{q_{1}+q_{2}+\mu_{i}} \Gamma\left(q_{1}+q_{2}+\mu_{i}+1\right)}\right) \\
& \left.+\Lambda_{2}\left(\sum_{l=1}^{r} \frac{\left|v_{l}\right|\left(\xi_{l}-a\right)^{q_{1}+q_{2}+\varphi_{l}}}{\rho^{q_{1}+q_{2}+\varphi_{l}} \Gamma\left(q_{1}+q_{2}+\varphi_{l}+1\right)}+\sum_{k=1}^{p} \frac{\left|\omega_{k}\right|\left(\psi_{k}-a\right)^{q_{1}+q_{2}+\gamma_{k}}}{\rho^{q_{1}+q_{2}+\gamma_{k}} \Gamma\left(q_{1}+q_{2}+\gamma_{k}+1\right)}\right)\right\} \\
& \times\left\|F_{x_{n}}-F_{x}\right\|_{\mathbb{E}} \\
& =\Lambda_{3}\left(q_{1}+q_{2}\right)\left\|F_{x_{n}}-F_{x}\right\|_{\mathbb{E}} \text {. }
\end{aligned}
$$

Since $f$ and $\lambda$ are continuous, by the Lebesgue dominated convergent theorem, we have

$$
\left|\left(\mathcal{Q}_{1} x_{n}\right)(t)-\left(\mathcal{Q}_{1} x\right)(t)\right| \rightarrow 0, \quad \text { as } n \rightarrow \infty .
$$

Hence,

$$
\left\|\mathcal{Q}_{1} x_{n}-\mathcal{Q}_{1} x\right\|_{\mathbb{E}} \rightarrow 0 \quad \text { as } n \rightarrow \infty
$$

Therefore, the operator $\mathcal{Q}_{1}$ is continuous. Also, the set $\mathcal{Q}_{1} B_{\bar{R}_{2}}$ is uniformly bounded as

$$
\left\|\mathcal{Q}_{1} x\right\|_{\mathbb{E}} \leq \Lambda_{3}\left(q_{1}+q_{2}\right)\|g\|_{\mathbb{E}}
$$

Next, we prove the compactness of the operator $\mathcal{Q}_{1}$. Set $\sup _{\left(t, z_{1}, z_{2}, z_{3}\right) \in[a, T] \times B_{\bar{R}_{2}}^{3}} \mid f\left(t, z_{1}, z_{2}\right.$, $\left.z_{3}\right) \mid=f^{*}<\infty$, then for each $t_{1}, t_{2} \in[a, T]$ with $a \leq t_{1} \leq t_{2} \leq T$, we have

$$
\begin{aligned}
& \left|\left(\mathcal{Q}_{1} x\right)\left(t_{2}\right)-\left(\mathcal{Q}_{1} x\right)\left(t_{1}\right)\right| \\
& \leq\left|a^{I^{q_{1}+q_{2}, \rho}} F_{x}(s)\left(t_{2}\right)-{ }_{a} I^{q_{1}+q_{2}, \rho} F_{x}(s)\left(t_{1}\right)\right|+\frac{1}{|\Omega|}\left|e^{\frac{\rho-1}{\rho}\left(t_{2}-a\right)}-e^{\frac{\rho-1}{\rho}\left(t_{1}-a\right)}\right| \\
& \quad \times\left[\left|\Omega_{4}\right|\left(\frac{\left(t_{2}-a\right)^{q_{2}}-\left(t_{1}-a\right)^{q_{2}}}{\rho^{q_{2}} \Gamma\left(q_{2}+1\right)}\right)\right. \\
& \quad \times\left(\sum_{j=1}^{n}\left|\alpha_{j}\right|_{a} I^{q_{1}+q_{2}+\beta_{j}, \rho} F_{x}(s)\left(\eta_{j}\right)+\sum_{i=1}^{m}\left|\kappa_{i}\right|_{a} I^{q_{1}+q_{2}+\mu_{i}, \rho} F_{x}(s)\left(\sigma_{i}\right)\right) \\
& \quad+\left|\Omega_{2}\right|\left(\frac{\left(t_{2}-a\right)^{q_{2}}-\left(t_{1}-a\right)^{q_{2}}}{\rho^{q_{2}} \Gamma\left(q_{2}+1\right)}\right) \\
& \left.\quad \times\left(\sum_{l=1}^{r}\left|v_{l}\right|_{a} I^{q_{1}+q_{2}+\varphi_{l}, \rho} F_{x}(s)\left(\xi_{l}\right)+\sum_{k=1}^{p}\left|\omega_{k}\right|_{a} I^{q_{1}+q_{2}+\gamma_{k}, \rho} F_{x}(s)\left(\psi_{k}\right)\right)\right]
\end{aligned}
$$




$$
\begin{aligned}
\leq & f^{*}\left\{\frac { 1 } { \rho ^ { q _ { 1 } + q _ { 2 } } \Gamma ( q _ { 1 } + q _ { 2 } + 1 ) } \left(\left|\left(t_{2}-a\right)^{q_{1}+q_{2}}-\left(t_{1}-a\right)^{q_{1}+q_{2}}-\left(t_{2}-t_{1}\right)^{q_{1}+q_{2}}\right|\right.\right. \\
& \left.+\left(t_{2}-t_{1}\right)^{q_{1}+q_{2}}\right)+\frac{1}{|\Omega|} \mid e^{\frac{\rho-1}{\rho}\left(t_{2}-a\right)} \\
& -e^{\frac{\rho-1}{\rho}\left(t_{1}-a\right)} \mid\left[| \Omega _ { 4 } | ( \frac { ( t _ { 2 } - a ) ^ { q _ { 2 } } - ( t _ { 1 } - a ) ^ { q _ { 2 } } } { \rho ^ { q _ { 2 } } \Gamma ( q _ { 2 } + 1 ) } ) \left(\sum_{j=1}^{n} \frac{\left|\alpha_{j}\right|\left(\eta_{j}-a\right)^{q_{1}+q_{2}+\beta_{j}}}{\rho^{q_{1}+q_{2}+\beta_{j}} \Gamma\left(q_{1}+q_{2}+\beta_{j}+1\right)}\right.\right. \\
& \left.+\sum_{i=1}^{m} \frac{\left|\kappa_{i}\right|\left(\sigma_{i}-a\right)^{q_{1}+q_{2}+\mu_{i}}}{\rho^{q_{1}+q_{2}+\mu_{i}} \Gamma\left(q_{1}+q_{2}+\mu_{i}+1\right)}\right)+\left|\Omega_{2}\right|\left(\frac{\left(t_{2}-a\right)^{q_{2}}-\left(t_{1}-a\right)^{q_{2}}}{\rho^{q_{2}} \Gamma\left(q_{2}+1\right)}\right) \\
& \left.\left.\times\left(\sum_{l=1}^{r} \frac{\left|v_{l}\right|\left(\xi_{l}-a\right)^{q_{1}+q_{2}+\varphi_{l}}}{\rho^{q_{1}+q_{2}+\varphi_{l}} \Gamma\left(q_{1}+q_{2}+\varphi_{l}+1\right)}+\sum_{k=1}^{p} \frac{\left|\omega_{k}\right|\left(\psi_{k}-a\right)^{q_{1}+q_{2}+\gamma_{k}}}{\rho^{q_{1}+q_{2}+\gamma_{k}} \Gamma\left(q_{1}+q_{2}+\gamma_{k}+1\right)}\right)\right]\right\},
\end{aligned}
$$

which is independent of $x$ and $\left|\left(\mathcal{Q}_{1} x\right)\left(t_{2}\right)-\left(\mathcal{Q}_{1} x\right)\left(t_{1}\right)\right| \rightarrow 0$ as $t_{2} \rightarrow t_{1}$. Therefore, the set $\mathcal{Q}_{1} B_{\bar{R}_{2}}$ is equicontinuous, the operator $\mathcal{Q}_{1}$ maps bounded subsets into relatively compact subsets, it follows that the set $\mathcal{Q}_{1} B_{\bar{R}_{2}}$ is relatively compact. Then, by the Arzelá-Ascoli theorem, the operator $\mathcal{Q}_{1}$ is compact on $B_{\bar{R}_{2}}$. It is easy to see that using $\Lambda_{4}<1$ leads to the operator $\mathcal{Q}_{2}$ is a contraction mapping and also assumption (iii) of Lemma 2.6 holds. Thus, all the assumptions of Lemma 2.6 are satisfied. Hence, the conclusion of Theorem 3.2 implies that problem (1.1) has at least one solution on $[a, T]$. This completes the proof.

\subsection{Existence result via Schaefer's fixed point theorem}

The last existence result is based on Schaefer's fixed point theorem.

Theorem 3.3 Assume that $\left(H_{1}\right),\left(H_{2}\right),\left(H_{4}\right)$, and $\left(H_{6}\right)$ hold. Then problem $(1.1)$ has at least one solution on $[a, T]$.

Proof To show that the operator $\mathcal{Q}$ has at least a fixed point in $\mathbb{E}$, the proof is divided into a sequence of four steps.

Step I The operator $\mathcal{Q}$ is continuous.

Let $x_{n}$ be a sequence such that $x_{n} \rightarrow x$ in $\mathbb{E}$. Then, for each $t \in[a, T]$, we obtain

$$
\begin{aligned}
& \left|\left(\mathcal{Q} x_{n}\right)(t)-(\mathcal{Q} x)(t)\right| \\
& \leq{ }_{a} I^{q_{1}+q_{2}, \rho}\left|F_{x_{n}}(s)-F_{x}(s)\right|(t)+{ }_{a} I^{q_{2}, \rho}|\lambda(s)|\left|x_{n}(s)-x(s)\right|(t) \\
& +\frac{e^{\frac{\rho-1}{\rho}(t-a)}}{|\Omega|}\left[( \frac { | \Omega _ { 4 } | ( t - a ) ^ { q _ { 2 } } } { \rho ^ { q _ { 2 } } \Gamma ( q _ { 2 } + 1 ) } + | \Omega _ { 3 } | ) \left(\sum _ { j = 1 } ^ { n } | \alpha _ { j } | \left[a^{I^{q_{1}+q_{2}+\beta_{j}, \rho}}\left|F_{x_{n}}(s)-F_{x}(s)\right|\left(\eta_{j}\right)\right.\right.\right. \\
& \left.+{ }_{a} I^{q_{2}+\beta_{j}, \rho}|\lambda(s)|\left|x_{n}(s)-x(s)\right|\left(\eta_{j}\right)\right]+\sum_{i=1}^{m}\left|\kappa_{i}\right|\left[I_{a} I^{q_{1}+q_{2}+\mu_{i}, \rho}\left|F_{x_{n}}(s)-F_{x}(s)\right|\left(\sigma_{i}\right)\right. \\
& \left.\left.+{ }_{a} I^{q_{2}+\mu_{i}, \rho}|\lambda(s)|\left|x_{n}(s)-x(s)\right|\left(\sigma_{i}\right)\right]\right)+\left(\left|\Omega_{1}\right|+\frac{\left|\Omega_{2}\right|(t-a)^{q_{2}}}{\rho^{q_{2}} \Gamma\left(q_{2}+1\right)}\right) \\
& \times\left(\sum_{l=1}^{r}\left|v_{l}\right|\left[I^{q^{q_{1}+q_{2}+\varphi_{l}, \rho}}\left|F_{x_{n}}(s)-F_{x}(s)\right|\left(\xi_{l}\right)+{ }_{a} I^{q_{2}+\varphi_{l}, \rho}|\lambda(s)|\left|x_{n}(s)-x(s)\right|\left(\xi_{l}\right)\right]\right. \\
& \left.\left.+\sum_{k=1}^{p}\left|\omega_{k}\right|\left[{ }_{a} I^{q_{1}+q_{2}+\gamma_{k}, \rho}\left|F_{x_{n}}(s)-F_{x}(s)\right|\left(\psi_{k}\right)+{ }_{a} I^{q_{2}+\gamma_{k}, \rho}|\lambda(s)|\left|x_{n}(s)-x(s)\right|\left(\psi_{k}\right)\right]\right)\right]
\end{aligned}
$$




$$
\begin{aligned}
\leq & {\left[\frac{(T-a)^{q_{1}+q_{2}}}{\rho^{q_{1}+q_{2}} \Gamma\left(q_{1}+q_{2}+1\right)}\right.} \\
& +\Lambda_{1}\left(\sum_{j=1}^{n} \frac{\left|\alpha_{j}\right|\left(\eta_{j}-a\right)^{q_{1}+q_{2}+\beta_{j}}}{\rho^{q_{1}+q_{2}+\beta_{j}} \Gamma\left(q_{1}+q_{2}+\beta_{j}+1\right)}+\sum_{i=1}^{m} \frac{\left|\kappa_{i}\right|\left(\sigma_{i}-a\right)^{q_{1}+q_{2}+\mu_{i}}}{\rho^{q_{1}+q_{2}+\mu_{i}} \Gamma\left(q_{1}+q_{2}+\mu_{i}+1\right)}\right) \\
& \left.+\Lambda_{2}\left(\sum_{l=1}^{r} \frac{\left|\nu_{l}\right|\left(\xi_{l}-a\right)^{q_{1}+q_{2}+\varphi_{l}}}{\rho^{q_{1}+q_{2}+\varphi_{l}} \Gamma\left(q_{1}+q_{2}+\varphi_{l}+1\right)}+\sum_{k=1}^{p} \frac{\left|\omega_{k}\right|\left(\psi_{k}-a\right)^{q_{1}+q_{2}+\gamma_{k}}}{\rho^{q_{1}+q_{2}+\gamma_{k}} \Gamma\left(q_{1}+q_{2}+\gamma_{k}+1\right)}\right)\right] \\
& \times\left\|F_{x_{n}}-F_{x}\right\|_{\mathbb{E}} \\
& +\left[{ }_{a} I^{q_{2}, \rho}|\lambda(s)|(T)+\Lambda_{1}\left(\sum_{j=1}^{n}\left|\alpha_{j}\right|_{a} I^{q_{2}+\beta_{j}, \rho}|\lambda(s)|\left(\eta_{j}\right)+\sum_{i=1}^{m}\left|\kappa_{i}\right|_{a} I^{q_{2}+\mu_{i}, \rho}|\lambda(s)|\left(\sigma_{i}\right)\right)\right. \\
& \left.+\Lambda_{2}\left(\sum_{l=1}^{r}\left|v_{l}\right|_{a} I^{q_{2}+\varphi_{l}, \rho}|\lambda(s)|\left(\xi_{l}\right)+\sum_{k=1}^{p}\left|\omega_{k}\right|_{a} I^{q_{2}+\gamma_{k}, \rho}|\lambda(s)|\left(\psi_{k}\right)\right)\right]\left\|x_{n}-x\right\|_{\mathbb{E}} \\
= & \Lambda_{3}\left(q_{1}+q_{2}\right)\left\|F_{x_{n}}-F_{x}\right\|_{\mathbb{E}}+\Lambda_{4}\left\|x_{n}-x\right\|_{\mathbb{E}} .
\end{aligned}
$$

Since $f$ and $\lambda$ are continuous, this implies that the operator $\mathcal{Q}$ is also continuous. Hence, we obtain

$$
\left\|F_{x_{n}}-F_{x}\right\|_{\mathbb{E}} \rightarrow 0 \text { and }\left\|x_{n}-x\right\|_{\mathbb{E}} \rightarrow 0, \quad \text { as } n \rightarrow \infty .
$$

Step II The operator $\mathcal{Q}$ maps a bounded set into a bounded set in $\mathbb{E}$.

For $R_{3}>0$, there exists a constant $M_{3}>0$ such that, for each $x \in \bar{B}_{R_{3}}=\left\{x \in \mathbb{E}:\|x\|_{\mathbb{E}} \leq\right.$ $\left.R_{3}\right\}$, then $\|\mathcal{Q} x\|_{\mathbb{E}} \leq M_{3}$. Then, for any $t \in[a, T]$ and $x \in \bar{B}_{R_{3}}$, we have

$$
\begin{aligned}
\mid(\mathcal{Q} x) & (t) \mid \\
\leq & { }_{a} I^{q_{1}+q_{2}, \rho}\left|F_{x}(s)\right|(t)+{ }_{a} I^{q_{2}, \rho}|\lambda(s)||x(s)|(t) \\
& +\frac{e^{\frac{\rho-1}{\rho}(t-a)}}{|\Omega|}\left[\left(\frac{\left|\Omega_{4}\right|(t-a)^{q_{2}}}{\rho^{q_{2}} \Gamma\left(q_{2}+1\right)}+\left|\Omega_{3}\right|\right)\right. \\
& \times\left(\sum_{j=1}^{n}\left|\alpha_{j}\right|\left[{ }_{a} I^{q_{1}+q_{2}+\beta_{j}, \rho}\left|F_{x}(s)\right|\left(\eta_{j}\right)+{ }_{a} I^{q_{2}+\beta_{j}, \rho}|\lambda(s)||x(s)|\left(\eta_{j}\right)\right]\right. \\
& \left.+\sum_{i=1}^{m}\left|\kappa_{i}\right|\left[{ }_{a} I^{q_{1}+q_{2}+\mu_{i}, \rho}\left|F_{x}(s)\right|\left(\sigma_{i}\right)+{ }_{a} I^{q_{2}+\mu_{i}, \rho}|\lambda(s)||x(s)|\left(\sigma_{i}\right)\right]\right) \\
& +\left(\left|\Omega_{1}\right|+\frac{\left|\Omega_{2}\right|(t-a)^{q_{2}}}{\rho^{q_{2}} \Gamma\left(q_{2}+1\right)}\right) \\
& \times\left(\sum_{l=1}^{r}\left|\nu_{l}\right|\left[{ }_{a} I^{q_{1}+q_{2}+\varphi_{l}, \rho}\left|F_{x}(s)\right|\left(\xi_{l}\right)+{ }_{a} I^{q_{2}+\varphi_{l}, \rho}|\lambda(s)||x(s)|\left(\xi_{l}\right)\right]\right. \\
& \left.\left.+\sum_{k=1}^{p}\left|\omega_{k}\right|\left[{ }_{a} I^{q_{1}+q_{2}+\gamma_{k}, \rho}\left|F_{x}(s)\right|\left(\psi_{k}\right)+{ }_{a} I^{q_{2}+\gamma_{k}, \rho}|\lambda(s)||x(s)|\left(\psi_{k}\right)\right]\right)\right] .
\end{aligned}
$$


It follows from hypotheses $\left(H_{4}\right)$ and $\left(H_{6}\right)$ that

$$
\begin{aligned}
{ }_{a} I^{u, \rho}\left|F_{x}(s)\right|(z) & \leq{ }_{a} I^{u, \rho}\left(h_{1}(s)+h_{2}(s)|x(s)|+h_{3}(s)\left|x(\epsilon s)+h_{4}(s)\right|(\mathcal{K} x)(s) \mid\right)(z) \\
& \leq \frac{1}{\rho^{u} \Gamma(u)} \int_{a}^{z}(z-s)^{u-1}\left(h_{1}^{*}+h_{2}^{*} R_{3}+h_{3}^{*} R_{3}+h_{4}^{*} R_{3} \phi_{0}(s-a)\right) d s \\
& \leq\left(h_{1}^{*}+h_{2}^{*} R_{3}+h_{3}^{*} R_{3}\right) \frac{(z-a)^{u}}{\rho^{u} \Gamma(u+1)}+h_{4}^{*} R_{3} \phi_{0} \frac{(z-a)^{u+1}}{\rho^{u} \Gamma(u+2)},
\end{aligned}
$$

where $u=\left\{q_{1}+q_{2}, q_{1}+q_{2}+\mu_{i}, q_{1}+q_{2}+\beta_{j}, q_{1}+q_{2}+\varphi_{l}, q_{1}+q_{2}+\gamma_{k}\right\}$ and $z=\left\{t, T, \sigma_{i}, \eta_{j}, \xi_{l}, \psi_{k}\right\}$.

This implies that

$$
\begin{aligned}
& |(\mathcal{Q} x)(t)| \\
& \leq\left[\frac{(T-a)^{q_{1}+q_{2}}}{\rho^{q_{1}+q_{2}} \Gamma\left(q_{1}+q_{2}+1\right)}\right. \\
& +\Lambda_{1}\left(\sum_{j=1}^{n} \frac{\left|\alpha_{j}\right|\left(\eta_{j}-a\right)^{q_{1}+q_{2}+\beta_{j}}}{\rho^{q_{1}+q_{2}+\beta_{j}} \Gamma\left(q_{1}+q_{2}+\beta_{j}+1\right)}+\sum_{i=1}^{m} \frac{\left|\kappa_{i}\right|\left(\sigma_{i}-a\right)^{q_{1}+q_{2}+\mu_{i}}}{\rho^{q_{1}+q_{2}+\mu_{i}} \Gamma\left(q_{1}+q_{2}+\mu_{i}+1\right)}\right) \\
& \left.+\Lambda_{2}\left(\sum_{l=1}^{r} \frac{\left|v_{l}\right|\left(\xi_{l}-a\right)^{q_{1}+q_{2}+\varphi_{l}}}{\rho^{q_{1}+q_{2}+\varphi_{l}} \Gamma\left(q_{1}+q_{2}+\varphi_{l}+1\right)}+\sum_{k=1}^{p} \frac{\left|\omega_{k}\right|\left(\psi_{k}-a\right)^{q_{1}+q_{2}+\gamma_{k}}}{\rho^{q_{1}+q_{2}+\gamma_{k}} \Gamma\left(q_{1}+q_{2}+\gamma_{k}+1\right)}\right)\right] \\
& \times\left(h_{1}^{*}+h_{2}^{*} R_{3}+h_{3}^{*} R_{3}\right) \\
& +\left[\frac{(T-a)^{q_{1}+q_{2}+1}}{\rho^{q_{1}+q_{2}} \Gamma\left(q_{1}+q_{2}+2\right)}\right. \\
& +\Lambda_{1}\left(\sum_{j=1}^{n} \frac{\left|\alpha_{j}\right|\left(\eta_{j}-a\right)^{q_{1}+q_{2}+\beta_{j}+1}}{\rho^{q_{1}+q_{2}+\beta_{j}} \Gamma\left(q_{1}+q_{2}+\beta_{j}+2\right)}+\sum_{i=1}^{m} \frac{\left|\kappa_{i}\right|\left(\sigma_{i}-a\right)^{q_{1}+q_{2}+\mu_{i}+1}}{\rho^{q_{1}+q_{2}+\mu_{i}} \Gamma\left(q_{1}+q_{2}+\mu_{i}+2\right)}\right) \\
& \left.+\Lambda_{2}\left(\sum_{l=1}^{r} \frac{\left|\nu_{l}\right|\left(\xi_{l}-a\right)^{q_{1}+q_{2}+\varphi_{l}+1}}{\rho^{q_{1}+q_{2}+\varphi_{l}} \Gamma\left(q_{1}+q_{2}+\varphi_{l}+2\right)}+\sum_{k=1}^{p} \frac{\left|\omega_{k}\right|\left(\psi_{k}-a\right)^{q_{1}+q_{2}+\gamma_{k}+1}}{\rho^{q_{1}+q_{2}+\gamma_{k}} \Gamma\left(q_{1}+q_{2}+\gamma_{k}+2\right)}\right)\right] \\
& \times h_{4}^{*} R_{3} \phi_{0} \\
& +\left[{ }_{a} I^{q_{2}, \rho}|\lambda(s)|(T)+\Lambda_{1}\left(\sum_{j=1}^{n}\left|\alpha_{j}\right|_{a} I^{q_{2}+\beta_{j}, \rho}|\lambda(s)|\left(\eta_{j}\right)+\sum_{i=1}^{m}\left|\kappa_{i}\right|_{a} I^{q_{2}+\mu_{i}, \rho}|\lambda(s)|\left(\sigma_{i}\right)\right)\right. \\
& \left.+\Lambda_{2}\left(\sum_{l=1}^{r}\left|\nu_{l}\right|_{a} I^{q_{2}+\varphi_{l}, \rho}|\lambda(s)|\left(\xi_{l}\right)+\sum_{k=1}^{p}\left|\omega_{k}\right|_{a} I^{q_{2}+\gamma_{k}, \rho}|\lambda(s)|\left(\psi_{k}\right)\right)\right] R_{3} \\
& \leq \Lambda_{3}\left(q_{1}+q_{2}\right)\left(h_{1}^{*}+h_{2}^{*} R_{3}+h_{3}^{*} R_{3}\right)+\Lambda_{3}\left(q_{1}+q_{2}+1\right) h_{4}^{*} R_{3} \phi_{0}+\Lambda_{4} R_{3}:=M_{3} \text {, }
\end{aligned}
$$

we estimate

$$
\|\mathcal{Q} x\|_{\mathbb{E}} \leq \Lambda_{3}\left(q_{1}+q_{2}\right)\left(h_{1}^{*}+h_{2}^{*} R_{3}+h_{3}^{*} R_{3}\right)+\Lambda_{3}\left(q_{1}+q_{2}+1\right) h_{4}^{*} R_{3} \phi_{0}+\Lambda_{4} R_{3}:=M_{3},
$$

where $\Lambda_{1}, \Lambda_{2}, \Lambda_{3}(u), u=\left\{q_{1}+q_{2}, q_{1}+q_{2}+1\right\}$, and $\Lambda_{4}$ are given by (3.2), (3.3), (3.4), and (3.5), respectively.

Step III The operator $\mathcal{Q}$ maps a bounded set into an equicontinuous set of $\mathbb{E}$. 
For $a \leq t_{1}<t_{2} \leq T$ and $x \in \bar{B}_{R_{3}}$ where $\bar{B}_{R_{3}}$ is as defined in Step II, by using the property $f$ is bounded on the compact set $[a, T] \times \bar{B}_{R_{3}}$, we have

$$
\begin{aligned}
& \left|(\mathcal{Q} x)\left(t_{2}\right)-(\mathcal{Q} x)\left(t_{1}\right)\right| \\
& \leq\left|{ }_{a} I^{q_{1}+q_{2}, \rho} F_{x}(s)\left(t_{2}\right)-{ }_{a} I^{q_{1}+q_{2}, \rho} F_{x}(s)\left(t_{1}\right)\right|+\left|{ }_{a} I^{q_{2}, \rho} \lambda(s) x(s)\left(t_{2}\right)-{ }_{a} I^{q_{2}, \rho} \lambda(s) x(s)\left(t_{1}\right)\right| \\
& +\frac{1}{|\Omega|}\left|e^{\frac{\rho-1}{\rho}\left(t_{2}-a\right)}-e^{\frac{\rho-1}{\rho}\left(t_{1}-a\right)}\right| \\
& \times\left[| \Omega _ { 4 } | ( \frac { ( t _ { 2 } - a ) ^ { q _ { 2 } } - ( t _ { 1 } - a ) ^ { q _ { 2 } } } { \rho ^ { q _ { 2 } } \Gamma ( q _ { 2 } + 1 ) } ) \left(\sum _ { j = 1 } ^ { n } | \alpha _ { j } | \left[{ }_{a} I^{q_{1}+q_{2}+\beta_{j}, \rho}\left|F_{x}(s)\right|\left(\eta_{j}\right)\right.\right.\right. \\
& \left.+{ }_{a} I^{q_{2}+\beta_{j}, \rho}|\lambda(s)||x(s)|\left(\eta_{j}\right)\right] \\
& \left.+\sum_{i=1}^{m}\left|\kappa_{i}\right|\left[{ }_{a} I^{q_{1}+q_{2}+\mu_{i}, \rho}\left|F_{x}(s)\right|\left(\sigma_{i}\right)+{ }_{a} I^{q_{2}+\mu_{i}, \rho}|\lambda(s)||x(s)|\left(\sigma_{i}\right)\right]\right) \\
& +\left|\Omega_{2}\right|\left(\frac{\left(t_{2}-a\right)^{q_{2}}-\left(t_{1}-a\right)^{q_{2}}}{\rho^{q_{2}} \Gamma\left(q_{2}+1\right)}\right) \\
& \times\left(\sum_{l=1}^{r}\left|\nu_{l}\right|\left[{ }_{a} I^{q_{1}+q_{2}+\varphi_{l}, \rho} F_{x}(s)\left(\xi_{l}\right)+{ }_{a} I^{q_{2}+\varphi_{l}, \rho}|\lambda(s)||x(s)|\left(\xi_{l}\right)\right]\right. \\
& \left.\left.+\sum_{k=1}^{p}\left|\omega_{k}\right|\left[{ }_{a} I^{q_{1}+q_{2}+\gamma_{k}, \rho} F_{x}(s)\left(\psi_{k}\right)+{ }_{a} I^{q_{2}+\gamma_{k}, \rho}|\lambda(s)||x(s)|\left(\psi_{k}\right)\right]\right)\right] \\
& \leq \frac{1}{\rho^{q_{1}+q_{2}} \Gamma\left(q_{1}+q_{2}\right)} \int_{a}^{t_{1}}\left|e^{\frac{\rho-1}{\rho}\left(t_{2}-s\right)}\left(t_{2}-s\right)^{q_{1}+q_{2}-1}-e^{\frac{\rho-1}{\rho}\left(t_{1}-s\right)}\left(t_{1}-s\right)^{q_{1}+q_{2}-1}\right| \\
& \times\left(h_{1}^{*}+h_{2}^{*} R_{3}+h_{3}^{*} R_{3}+h_{4}^{*} R_{3} \phi_{0}(s-a)\right) d s \\
& +\frac{1}{\rho^{q_{1}+q_{2}} \Gamma\left(q_{1}+q_{2}\right)} \int_{t_{1}}^{t_{2}} e^{\frac{\rho-1}{\rho}\left(t_{2}-s\right)}\left(t_{2}-s\right)^{q_{1}+q_{2}-1} \\
& \times\left(h_{1}^{*}+h_{2}^{*} R_{3}+h_{3}^{*} R_{3}+h_{4}^{*} R_{3} \phi_{0}(s-a)\right) d s \\
& +\frac{R_{3}}{\rho^{q_{2}} \Gamma\left(q_{2}\right)} \int_{t_{1}}^{t_{2}} e^{\frac{\rho-1}{\rho}\left(t_{2}-s\right)}\left(t_{2}-s\right)^{q_{1}+q_{2}-1}|\lambda(s)| d s \\
& +\frac{R_{3}}{\rho^{q_{2}} \Gamma\left(q_{2}\right)} \int_{a}^{t_{1}}\left|e^{\frac{\rho-1}{\rho}\left(t_{2}-s\right)}\left(t_{2}-s\right)^{q_{1}+q_{2}-1}-e^{\frac{\rho-1}{\rho}\left(t_{1}-s\right)}\left(t_{1}-s\right)^{q_{1}+q_{2}-1}\right||\lambda(s)| d s \\
& +\frac{h_{1}^{*}+h_{2}^{*} R_{3}+h_{3}^{*} R_{3}+h_{4}^{*} R_{3} \phi_{0}}{|\Omega|}\left|e^{\frac{\rho-1}{\rho}\left(t_{2}-a\right)}-e^{\frac{\rho-1}{\rho}\left(t_{1}-a\right)}\right| \\
& \times\left[\left|\Omega_{4}\right|\left(\frac{\left(t_{2}-a\right)^{q_{2}}-\left(t_{1}-a\right)^{q_{2}}}{\rho^{q_{2}} \Gamma\left(q_{2}+1\right)}\right)\right. \\
& \times\left(\sum_{j=1}^{n}\left|\alpha_{j}\right|\left(\frac{\left(\eta_{j}-a\right)^{q_{1}+q_{2}+\beta_{j}}}{\rho^{q_{1}+q_{2}+\beta_{j}} \Gamma\left(q_{1}+q_{2}+\beta_{j}+1\right)}+\frac{\left(\eta_{j}-a\right)^{q_{1}+q_{2}+\beta_{j}+1}}{\rho^{q_{1}+q_{2}+\beta_{j}} \Gamma\left(q_{1}+q_{2}+\beta_{j}+2\right)}\right)\right. \\
& \left.+\sum_{i=1}^{m}\left|\kappa_{i}\right|\left(\frac{\left(\sigma_{i}-a\right)^{q_{1}+q_{2}+\mu_{i}}}{\rho^{q_{1}+q_{2}+\mu_{i}} \Gamma\left(q_{1}+q_{2}+\mu_{i}+1\right)}+\frac{\left(\sigma_{i}-a\right)^{q_{1}+q_{2}+\mu_{i}+1}}{\rho^{q_{1}+q_{2}+\mu_{i}} \Gamma\left(q_{1}+q_{2}+\mu_{i}+2\right)}\right)\right) \\
& +\left(\sum_{l=1}^{r}\left|v_{l}\right|\left(\frac{\left(\xi_{l}-a\right)^{q_{1}+q_{2}+\varphi_{l}}}{\rho^{q_{1}+q_{2}+\varphi_{l}} \Gamma\left(q_{1}+q_{2}+\varphi_{l}+1\right)}+\frac{\left(\xi_{l}-a\right)^{q_{1}+q_{2}+\varphi_{l}+1}}{\rho^{q_{1}+q_{2}+\varphi_{l}} \Gamma\left(q_{1}+q_{2}+\varphi_{l}+2\right)}\right)\right.
\end{aligned}
$$




$$
\begin{aligned}
& \left.+\sum_{k=1}^{p}\left|\omega_{k}\right|\left(\frac{\left(\psi_{k}-a\right)^{q_{1}+q_{2}+\gamma_{k}}}{\rho^{q_{1}+q_{2}+\gamma_{k}} \Gamma\left(q_{1}+q_{2}+\gamma_{k}+1\right)}+\frac{\left(\psi_{k}-a\right)^{q_{1}+q_{2}+\gamma_{k}+1}}{\rho^{q_{1}+q_{2}+\gamma_{k}} \Gamma\left(q_{1}+q_{2}+\gamma_{k}+2\right)}\right)\right) \\
& \left.\times\left|\Omega_{2}\right|\left(\frac{\left(t_{2}-a\right)^{q_{2}}-\left(t_{1}-a\right)^{q_{2}}}{\rho^{q_{2}} \Gamma\left(q_{2}+1\right)}\right)\right]+R_{3}\left[\sum_{j=1}^{n}\left|\alpha_{j}\right|_{a} I^{q_{2}+\beta_{j}, \rho}|\lambda(s)|\left(\eta_{j}\right)\right. \\
& +\sum_{i=1}^{m}\left|\kappa_{i}\right|_{a} I^{q_{2}+\mu_{i}, \rho}|\lambda(s)|\left(\sigma_{i}\right)+\sum_{l=1}^{r}\left|\nu_{l}\right|_{a} I^{q_{2}+\varphi_{l}, \rho}|\lambda(s)|\left(\xi_{l}\right) \\
& \left.+\sum_{k=1}^{p}\left|\omega_{k}\right|_{a} I^{q_{2}+\gamma_{k}, \rho}|\lambda(s)|\left(\psi_{k}\right)\right] .
\end{aligned}
$$

The R.H.S of the above inequality tends to zero as $t_{2} \rightarrow t_{1}$ implies that $\|(\mathcal{Q} x)\left(t_{2}\right)-$ $(\mathcal{Q} x)\left(t_{1}\right) \|_{\mathbb{E}} \rightarrow 0$ as $t_{2} \rightarrow t_{1}$. Thus, by Steps I to III, together with the Arzelá-Ascoli theorem, we conclude that the operator $\mathcal{Q}$ is completely continuous.

Step IV The set $\mathbb{D}=\{x \in \mathbb{E}: x=\varrho \mathcal{Q} x, 0 \leq \varrho \leq 1\}$ is bounded (a priori bounds).

Let $x \in \mathbb{D}$, then $x=\varrho \mathcal{Q} x$ for some $0<\varrho<1$. From $\left(H_{4}\right)-\left(H_{5}\right)$, for each $t \in[a, T]$, one can get the estimates

$$
\begin{aligned}
& |(\mathcal{Q} x)(t)| \\
& =|\varrho(\mathcal{Q} x)(t)| \\
& \leq{ }_{a} I^{q_{1}+q_{2}, \rho}\left|F_{x}(s)\right|(T)+{ }_{a} I^{q_{2}, \rho}|\lambda(s)||x(s)|(T)+\frac{e^{\frac{\rho-1}{\rho}(T-a)}}{|\Omega|}\left[\left(\frac{\left|\Omega_{4}\right|(T-a)^{q_{2}}}{\rho^{q_{2}} \Gamma\left(q_{2}+1\right)}+\left|\Omega_{3}\right|\right)\right. \\
& \times\left(\sum_{j=1}^{n}\left|\alpha_{j}\right|\left[{ }_{a} I^{q_{1}+q_{2}+\beta_{j}, \rho}\left|F_{x}(s)\right|\left(\eta_{j}\right)+{ }_{a} I^{q_{2}+\beta_{j}, \rho}|\lambda(s)||x(s)|\left(\eta_{j}\right)\right]\right. \\
& \left.+\sum_{i=1}^{m}\left|\kappa_{i}\right|\left[{ }_{a} I^{q_{1}+q_{2}+\mu_{i}, \rho}\left|F_{x}(s)\right|\left(\sigma_{i}\right)+{ }_{a} I^{q_{2}+\mu_{i}, \rho}|\lambda(s)||x(s)|\left(\sigma_{i}\right)\right]\right) \\
& +\left(\left|\Omega_{1}\right|+\frac{\left|\Omega_{2}\right|(T-a)^{q_{2}}}{\rho^{q_{2}} \Gamma\left(q_{2}+1\right)}\right)\left(\sum _ { l = 1 } ^ { r } | \nu _ { l } | \left[a_{a} I^{q_{1}+q_{2}+\varphi_{l}, \rho}\left|F_{x}(s)\right|\left(\xi_{l}\right)\right.\right. \\
& \left.+{ }_{a} I^{q_{2}+\varphi_{l}, \rho}|\lambda(s)||x(s)|\left(\xi_{l}\right)\right] \\
& \left.\left.+\sum_{k=1}^{p}\left|\omega_{k}\right|\left[{ }_{a} I^{q_{1}+q_{2}+\gamma_{k}, \rho}\left|F_{x}(s)\right|\left(\psi_{k}\right)+{ }_{a} I^{q_{2}+\gamma_{k}, \rho}|\lambda(s)||x(s)|\left(\psi_{k}\right)\right]\right)\right] \\
& \leq\left[\frac{(T-a)^{q_{1}+q_{2}}}{\rho^{q_{1}+q_{2}} \Gamma\left(q_{1}+q_{2}+1\right)}\right. \\
& +\Lambda_{1}\left(\sum_{j=1}^{n} \frac{\left|\alpha_{j}\right|\left(\eta_{j}-a\right)^{q_{1}+q_{2}+\beta_{j}}}{\rho^{q_{1}+q_{2}+\beta_{j}} \Gamma\left(q_{1}+q_{2}+\beta_{j}+1\right)}+\sum_{i=1}^{m} \frac{\left|\kappa_{i}\right|\left(\sigma_{i}-a\right)^{q_{1}+q_{2}+\mu_{i}}}{\rho^{q_{1}+q_{2}+\mu_{i}} \Gamma\left(q_{1}+q_{2}+\mu_{i}+1\right)}\right) \\
& \left.+\Lambda_{2}\left(\sum_{l=1}^{r} \frac{\left|\nu_{l}\right|\left(\xi_{l}-a\right)^{q_{1}+q_{2}+\varphi_{l}}}{\rho^{q_{1}+q_{2}+\varphi_{l}} \Gamma\left(q_{1}+q_{2}+\varphi_{l}+1\right)}+\sum_{k=1}^{p} \frac{\left|\omega_{k}\right|\left(\psi_{k}-a\right)^{q_{1}+q_{2}+\gamma_{k}}}{\rho^{q_{1}+q_{2}+\gamma_{k}} \Gamma\left(q_{1}+q_{2}+\gamma_{k}+1\right)}\right)\right] \\
& \times\left(h_{1}^{*}+h_{2}^{*} R_{3}+h_{3}^{*} R_{3}\right)
\end{aligned}
$$




$$
\begin{aligned}
& +\left[\frac{(T-a)^{q_{1}+q_{2}+1}}{\rho^{q_{1}+q_{2}} \Gamma\left(q_{1}+q_{2}+2\right)}\right. \\
& +\Lambda_{1}\left(\sum_{j=1}^{n} \frac{\left|\alpha_{j}\right|\left(\eta_{j}-a\right)^{q_{1}+q_{2}+\beta_{j}+1}}{\rho^{q_{1}+q_{2}+\beta_{j}} \Gamma\left(q_{1}+q_{2}+\beta_{j}+2\right)}+\sum_{i=1}^{m} \frac{\left|\kappa_{i}\right|\left(\sigma_{i}-a\right)^{q_{1}+q_{2}+\mu_{i}+1}}{\rho^{q_{1}+q_{2}+\mu_{i}} \Gamma\left(q_{1}+q_{2}+\mu_{i}+2\right)}\right) \\
& \left.+\Lambda_{2}\left(\sum_{l=1}^{r} \frac{\left|\nu_{l}\right|\left(\xi_{l}-a\right)^{q_{1}+q_{2}+\varphi_{l}+1}}{\rho^{q_{1}+q_{2}+\varphi_{l}} \Gamma\left(q_{1}+q_{2}+\varphi_{l}+2\right)}+\sum_{k=1}^{p} \frac{\left|\omega_{k}\right|\left(\psi_{k}-a\right)^{q_{1}+q_{2}+\gamma_{k}+1}}{\rho^{q_{1}+q_{2}+\gamma_{k}} \Gamma\left(q_{1}+q_{2}+\gamma_{k}+2\right)}\right)\right] \\
& \times h_{4}^{*} R_{3} \phi_{0} \\
& +\left[{ }_{a} I^{q_{2}, \rho}|\lambda(s)|(T)+\Lambda_{1}\left(\sum_{j=1}^{n}\left|\alpha_{j}\right|_{a} I^{q_{2}+\beta_{j}, \rho}|\lambda(s)|\left(\eta_{j}\right)+\sum_{i=1}^{m}\left|\kappa_{i}\right|_{a} I^{q_{2}+\mu_{i}, \rho}|\lambda(s)|\left(\sigma_{i}\right)\right)\right. \\
& \left.+\Lambda_{2}\left(\sum_{l=1}^{r}\left|\nu_{l}\right|_{a} I^{q_{2}+\varphi_{l}, \rho}|\lambda(s)|\left(\xi_{l}\right)+\sum_{k=1}^{p}\left|\omega_{k}\right|_{a} I^{q_{2}+\gamma_{k}, \rho}|\lambda(s)|\left(\psi_{k}\right)\right)\right] R_{3} \\
& =\Lambda_{3}\left(q_{1}+q_{2}\right)\left(h_{1}^{*}+h_{2}^{*} R_{3}+h_{3}^{*} R_{3}\right)+\Lambda_{3}\left(q_{1}+q_{2}+1\right) h_{4}^{*} R_{3} \phi_{0}+\Lambda_{4} R_{3} .
\end{aligned}
$$

Thus, $\|\mathcal{Q} x\|_{\mathbb{E}} \leq \Lambda_{3}\left(q_{1}+q_{2}\right)\left(h_{1}^{*}+h_{2}^{*} R_{3}+h_{3}^{*} R_{3}\right)+\Lambda_{3}\left(q_{1}+q_{2}+1\right) h_{4}^{*} R_{3} \phi_{0}+\Lambda_{4} R_{3}:=N<\infty$. This implies that the set $\mathbb{D}$ is bounded.

By all the hypotheses of Theorem 3.3, we conclude that there exists a positive constant $N$ such that $\|x\|_{\mathbb{E}} \leq N<\infty$. By applying Schaefer's fixed point theorem (Lemma 2.7), the operator $\mathcal{Q}$ has at least one fixed point which is a solution of problem (1.1). This completes the proof.

\section{Ulam-Hyers stability results}

In this section, we investigate the Ulam stability of problem (1.1), namely Ulam-Hyers stable, generalized Ulam-Hyers stable, Ulam-Hyers-Rassias stable, and generalized UlamHyers-Rassias stable.

Definition 4.1 ([39]) Problem (1.1) is said to be Ulam-Hyers stable if there exists $\Phi \in$ $\mathbb{R}^{+} \backslash\{0\}$ such that, for each $\varrho>0$ and solution $z \in \mathbb{E}^{1}=C^{1}([a, T], \mathbb{R})$ of the inequality

$$
\left|{ }_{a}^{C} D^{q_{1}, \rho}\left({ }_{a}^{C} D^{q_{2}, \rho}+\lambda(t)\right) z(t)-f(t, z(t), z(\theta(t)),(\mathcal{K} z)(t))\right| \leq \varrho, \quad t \in[a, T],
$$

there exists a solution $x \in \mathbb{E}^{1}$ of problem (1.1) such that

$$
|z(t)-x(t)| \leq \Phi \varrho, \quad t \in[a, T] .
$$

Definition 4.2 ([39]) Problem (1.1) is said to be generalized Ulam-Hyers stable if there exists $\Phi_{f} \in C\left(\mathbb{R}^{+}, \mathbb{R}^{+}\right)$with $\Phi_{f}(0)=0$ such that, for each solution $z \in \mathbb{E}^{1}$ of inequality (4.1), there exists a solution $x \in \mathbb{E}^{1}$ of problem (1.1) such that

$$
|z(t)-x(t)| \leq \Phi_{f} \varrho, \quad t \in[a, T] .
$$

Definition 4.3 ([39]) Problem (1.1) is said to be Ulam-Hyers-Rassias stable with respect to $\Phi_{f} \in C\left([a, T], \mathbb{R}^{+}\right)$if there exists a real number $C_{f, \Phi}>0$ such that, for $\varrho>0$ and for each 
solution $z \in \mathbb{E}^{1}$ of the inequality

$$
\left|{ }_{a}^{C} D^{q_{1}, \rho}\left({ }_{a}^{C} D^{q_{2}, \rho}+\lambda(t)\right) z(t)-f(t, z(t), z(\theta(t)),(\mathcal{K} z)(t))\right| \leq \varrho \Phi_{f}(t), \quad t \in[a, T],
$$

there exists a solution $x \in \mathbb{E}^{1}$ of problem (1.1) such that

$$
|z(t)-x(t)| \leq C_{f, \Phi} \varrho \Phi_{f}(t), \quad t \in[a, T] .
$$

Definition 4.4 ([39]) Problem (1.1) is said to be generalized Ulam-Hyers-Rassias stable with respect to $\Phi_{f} \in C\left([a, T], \mathbb{R}^{+}\right)$if there exists a real number $C_{f, \Phi}>0$ such that, for each solution $z \in \mathbb{E}^{1}$ of the inequality

$$
\left|{ }_{a}^{C} D^{q_{1}, \rho}\left({ }_{a}^{C} D^{q_{2}, \rho}+\lambda(t)\right) z(t)-f(t, z(t), z(\theta(t)),(\mathcal{K} z)(t))\right| \leq \Phi_{f}(t), \quad t \in[a, T],
$$

there exists a solution $x \in \mathbb{E}^{1}$ of problem (1.1) such that

$$
|z(t)-x(t)| \leq C_{f, \Phi} \Phi_{f}(t), \quad t \in[a, T] .
$$

Remark 4.5 It is clear that

(i) Definition $4.1 \Rightarrow$ Definition 4.2;

(ii) Definition $4.3 \Rightarrow$ Definition 4.4 ;

(iii) Definition 4.3 for $\Phi_{f}(\cdot)=1 \Rightarrow$ Definition 4.1.

Remark 4.6 A function $z \in \mathbb{E}^{1}$ is a solution of inequality (4.1) if and only if there exists a function $\Psi \in C([a, T], \mathbb{R})$ (dependent on $z$ ) such that

(i) $|\Psi(t)| \leq \varrho, \forall t \in[a, T]$;

(ii) ${ }_{a}^{C} D^{q_{1}, \rho}\left({ }_{a}^{C} D^{q_{2}, \rho}+\lambda(t)\right) z(t)=f(t, z(t), z(\theta(t)),(\mathcal{K} z)(t))+\Psi(t), t \in[a, T]$.

By Remark 4.6, the solution of the problem

$$
{ }_{a}^{C} D^{q_{1}, \rho}\left({ }_{a}^{C} D^{q_{2}, \rho}+\lambda(t)\right) z(t)=f(t, z(t), z(\theta(t)),(\mathcal{K} z)(t))+\Psi(t), \quad t \in[a, T],
$$

can be written as follows:

$$
\begin{aligned}
& z(t)={ }_{a}{ }^{q_{1}+q_{2}, \rho} F_{z}(s)(t)-{ }_{a} I^{q_{2}, \rho} \lambda(s) x(s)(t) \\
& +\frac{e^{\frac{\rho-1}{\rho}(t-a)}}{\Omega}\left[\left(\frac{\Omega_{4}(t-a)^{q_{2}}}{\rho^{q_{2}} \Gamma\left(q_{2}+1\right)}-\Omega_{3}\right)\right. \\
& \times\left(\sum_{j=1}^{n} \alpha_{j}\left[I^{I^{q_{1}+q_{2}+\beta_{j}, \rho}} F_{x}(s)\left(\eta_{j}\right)-{ }_{a} I^{q_{2}+\beta_{j}, \rho} \lambda(s) x(s)\left(\eta_{j}\right)\right]\right. \\
& \left.-\sum_{i=1}^{m} \kappa_{i}\left[a^{q^{q_{1}+q_{2}+\mu_{i}, \rho}} F_{x}(s)\left(\sigma_{i}\right)-{ }_{a} I^{q_{2}+\mu_{i}, \rho} \lambda(s) x(s)\left(\sigma_{i}\right)\right]\right) \\
& +\left(\Omega_{1}-\frac{\Omega_{2}(t-a)^{q_{2}}}{\rho^{q_{2}} \Gamma\left(q_{2}+1\right)}\right)\left(\sum_{l=1}^{r} \nu_{l}\left[I^{q_{1}+q_{2}+\varphi_{l}, \rho} F_{x}(s)\left(\xi_{l}\right)-{ }_{a} I^{q_{2}+\varphi_{l}, \rho} \lambda(s) x(s)\left(\xi_{l}\right)\right]\right.
\end{aligned}
$$




$$
\begin{aligned}
& \left.\left.-\sum_{k=1}^{p} \omega_{k}\left[a^{I^{q_{1}+q_{2}+\gamma_{k}, \rho}} F_{x}(s)\left(\psi_{k}\right)-{ }_{a} I^{q_{2}+\gamma_{k}, \rho} \lambda(s) x(s)\left(\psi_{k}\right)\right]\right)\right]+{ }_{a} I^{q_{1}+q_{2}, \rho} \Psi(s)(t) \\
& +\frac{e^{\frac{\rho-1}{\rho}(t-a)}}{\Omega}\left[\left(\frac{\Omega_{4}(t-a)^{q_{2}}}{\rho^{q_{2}} \Gamma\left(q_{2}+1\right)}-\Omega_{3}\right)\right. \\
& \times\left(\sum_{j=1}^{n} \alpha_{j a} I^{q_{1}+q_{2}+\beta_{j}, \rho} \Psi(s)\left(\eta_{j}\right)-\sum_{i=1}^{m} \kappa_{i a} I^{q_{1}+q_{2}+\mu_{i}, \rho} \Psi(s)\left(\sigma_{i}\right)\right) \\
& +\left(\Omega_{1}-\frac{\Omega_{2}(t-a)^{q_{2}}}{\rho^{q_{2}} \Gamma\left(q_{2}+1\right)}\right) \\
& \left.\times\left(\sum_{l=1}^{r} v_{l a} I^{q_{1}+q_{2}+\varphi_{l}, \rho} \Psi(s)\left(\xi_{l}\right)-\sum_{k=1}^{p} \omega_{k a} I^{q_{1}+q_{2}+\gamma_{k}, \rho} \Psi(s)\left(\psi_{k}\right)\right)\right] .
\end{aligned}
$$

Firstly, we present an important lemma that will be used in the proofs of the first stability theorem.

Lemma 4.7 If $z \in \mathbb{E}^{1}$ satisfies inequality (4.1), then the function $z$ is a solution of the following inequality:

$$
|z(t)-(\mathcal{Q} z)(t)| \leq \Lambda_{3}\left(q_{1}+q_{2}\right) \varrho, \quad 0<\varrho \leq 1,
$$

where $\Lambda_{3}\left(q_{1}+q_{2}\right)$ is given by (3.4).

Proof From Remark 4.6 with (4.8), we obtain

$$
\begin{aligned}
\mid z(t) & -(\mathcal{Q} z)(t) \mid \\
= & \mid a^{q_{1}+q_{2}, \rho} \Psi(s)(t)+\frac{e^{\frac{\rho-1}{\rho}(t-a)}}{\Omega}\left[( \frac { \Omega _ { 4 } ( t - a ) ^ { q _ { 2 } } } { \rho ^ { q _ { 2 } } \Gamma ( q _ { 2 } + 1 ) } - \Omega _ { 3 } ) \left(\sum_{j=1}^{n} \alpha_{j a} I^{q_{1}+q_{2}+\beta_{j}, \rho} \Psi(s)\left(\eta_{j}\right)\right.\right. \\
& \left.-\sum_{i=1}^{m} \kappa_{i a} I^{q_{1}+q_{2}+\mu_{i}, \rho} \Psi(s)\left(\sigma_{i}\right)\right)+\left(\Omega_{1}-\frac{\Omega_{2}(t-a)^{q_{2}}}{\rho^{q_{2}} \Gamma\left(q_{2}+1\right)}\right)\left(\sum_{l=1}^{r} v_{l a} I^{q_{1}+q_{2}+\varphi_{l}, \rho} \Psi(s)\left(\xi_{l}\right)\right. \\
& \left.\left.-\sum_{k=1}^{p} \omega_{k a} I^{q_{1}+q_{2}+\gamma_{k}, \rho} \Psi(s)\left(\psi_{k}\right)\right)\right] \mid \\
\leq & \left\{\frac{(T-a)^{q_{1}+q_{2}}}{\rho^{q_{1}+q_{2}} \Gamma\left(q_{1}+q_{2}+1\right)}\right. \\
& +\Lambda_{1}\left(\sum_{j=1}^{n} \frac{\left|\alpha_{j}\right|\left(\eta_{j}-a\right)^{q_{1}+q_{2}+\beta_{j}}}{\rho^{q_{1}+q_{2}+\beta_{j}} \Gamma\left(q_{1}+q_{2}+\beta_{j}+1\right)}+\sum_{i=1}^{m} \frac{\left|\kappa_{i}\right|\left(\sigma_{i}-a\right)^{q_{1}+q_{2}+\mu_{i}}}{\rho^{q_{1}+q_{2}+\mu_{i}} \Gamma\left(q_{1}+q_{2}+\mu_{i}+1\right)}\right) \\
& \left.+\Lambda_{2}\left(\sum_{l=1}^{r} \frac{\left|\nu_{l}\right|\left(\xi_{l}-a\right)^{q_{1}+q_{2}+\varphi_{l}}}{\rho^{q_{1}+q_{2}+\varphi_{l}} \Gamma\left(q_{1}+q_{2}+\varphi_{l}+1\right)}+\sum_{k=1}^{p} \frac{\left|\omega_{k}\right|\left(\psi_{k}-a\right)^{q_{1}+q_{2}+\gamma_{k}}}{\rho^{q_{1}+q_{2}+\gamma_{k}} \Gamma\left(q_{1}+q_{2}+\gamma_{k}+1\right)}\right)\right\} \\
= & \Lambda_{3}\left(q_{1}+q_{2}\right) \varrho,
\end{aligned}
$$

where $\Lambda_{3}\left(q_{1}+q_{2}\right)$ is given by (3.4), from which inequality (4.9) is obtained.

Now, we present the Ulam-Hyers stability and generalized Ulam-Hyers stability results. 
Theorem 4.8 Assume that $\left(H_{1}\right),\left(H_{2}\right),\left(H_{3}\right),\left(H_{4}\right)$ are satisfied with

$$
2 L_{1} \Lambda_{3}\left(q_{1}+q_{2}\right)+L_{2} \phi_{0} \Lambda_{3}\left(q_{1}+q_{2}+1\right)+\Lambda_{4}<1 \text {, }
$$

where $\Lambda_{3}(u), u=\left\{q_{1}+q_{2}, q_{1}+q_{2}+1\right\}, \Lambda_{4}$ are defined by (3.4) and (3.5), respectively. Then problem (1.1) is both Ulam-Hyers stable and generalized Ulam-Hyers stable on $[a, T]$.

Proof Let $z \in \mathbb{E}^{1}$ be a solution of inequality (4.1), and let $x$ be the unique solution of problem (1.1),

$$
\left\{\begin{array}{l}
{ }_{a}^{C} D^{q_{1}, \rho}\left({ }_{a}^{C} D^{q_{2}, \rho}+\lambda(t)\right) x(t)=f(t, x(t), x(\theta(t)),(\mathcal{K} x)(t)), \quad t \in[a, T] \\
\sum_{i=1}^{m} \kappa_{i a} I^{\mu_{i}, \rho} x\left(\sigma_{i}\right)=\sum_{j=1}^{n} \alpha_{j a} I^{\beta_{j}, \rho} x\left(\eta_{j}\right), \quad \sum_{k=1}^{p} \omega_{k a} I^{\gamma_{k}, \rho} x\left(\psi_{k}\right)=\sum_{l=1}^{r} v_{l a} I^{\varphi_{l}, \rho} x\left(\xi_{l}\right) .
\end{array}\right.
$$

By applying the triangle inequality, $|u-v| \leq|u|+|v|$, and Lemma 4.7, we have

$$
\begin{aligned}
& |z(t)-x(t)| \\
& =\mid z(t)-\left\{{ }_{a} I^{q_{1}+q_{2}, \rho} F_{x}(s)(t)-{ }_{a} I^{q_{2}, \rho} \lambda(s) x(s)(t)\right. \\
& +\frac{e^{\frac{\rho-1}{\rho}(t-a)}}{\Omega}\left[\left(\frac{\Omega_{4}(t-a)^{q_{2}}}{\rho^{q_{2}} \Gamma\left(q_{2}+1\right)}-\Omega_{3}\right)\right. \\
& \times\left(\sum_{j=1}^{n} \alpha_{j}\left[{ }_{a} I^{q_{1}+q_{2}+\beta_{j}, \rho} F_{x}(s)\left(\eta_{j}\right)-{ }_{a} I^{q_{2}+\beta_{j}, \rho} \lambda(s) x(s)\left(\eta_{j}\right)\right]\right. \\
& \left.-\sum_{i=1}^{m} \kappa_{i}\left[{ }_{a} I^{q_{1}+q_{2}+\mu_{i}, \rho} F_{x}(s)\left(\sigma_{i}\right)-{ }_{a} I^{q_{2}+\mu_{i}, \rho} \lambda(s) x(s)\left(\sigma_{i}\right)\right]\right) \\
& +\left(\Omega_{1}-\frac{\Omega_{2}(t-a)^{q_{2}}}{\rho^{q_{2}} \Gamma\left(q_{2}+1\right)}\right)\left(\sum_{l=1}^{r} v_{l}\left[{ }_{a} I^{q_{1}+q_{2}+\varphi_{l}, \rho} F_{x}(s)\left(\xi_{l}\right)-{ }_{a} I^{q_{2}+\varphi_{l}, \rho} \lambda(s) x(s)\left(\xi_{l}\right)\right]\right. \\
& \left.\left.\left.-\sum_{k=1}^{p} \omega_{k}\left[I^{q_{1}+q_{2}+\gamma_{k}, \rho} F_{x}(s)\left(\psi_{k}\right)-{ }_{a} I^{q_{2}+\gamma_{k}, \rho} \lambda(s) x(s)\left(\psi_{k}\right)\right]\right)\right]\right\} \mid \\
& =|z(t)-(\mathcal{Q} z)(t)+(\mathcal{Q} z)(t)-(\mathcal{Q} x)(t)| \\
& \leq|z(t)-(\mathcal{Q} z)(t)|+|(\mathcal{Q} z)(t)-(\mathcal{Q} x)(t)| \\
& \leq \Lambda_{3}\left(q_{1}+q_{2}\right) \varrho+\left[2 L_{1} \Lambda_{3}\left(q_{1}+q_{2}\right)+L_{2} \phi_{0} \Lambda_{3}\left(q_{1}+q_{2}+1\right)+\Lambda_{4}\right]|z(t)-x(t)|,
\end{aligned}
$$

where $\Lambda_{3}(u), u=\left\{q_{1}+q_{2}, q_{1}+q_{2}+1\right\}$, and $\Lambda_{4}$ are defined by (3.4) and (3.5), respectively. This yields that

$$
|z(t)-x(t)| \leq \frac{\Lambda_{3}\left(q_{1}+q_{2}\right) \varrho}{1-\left[2 L_{1} \Lambda_{3}\left(q_{1}+q_{2}\right)+L_{2} \phi_{0} \Lambda_{3}\left(q_{1}+q_{2}+1\right)+\Lambda_{4}\right]} .
$$

By setting

$$
\Phi:=\frac{\Lambda_{3}\left(q_{1}+q_{2}\right)}{1-\left[2 L_{1} \Lambda_{3}\left(q_{1}+q_{2}\right)+L_{2} \phi_{0} \Lambda_{3}\left(q_{1}+q_{2}+1\right)+\Lambda_{4}\right]},
$$


we end up with

$$
|z(t)-x(t)| \leq \Phi \varrho .
$$

Hence, problem (1.1) is Ulam-Hyers stable. Moreover, if we set $\Phi_{f}(\varrho)=\Phi \varrho$ such that $\Phi_{f}(0)=0$, then problem (1.1) is generalized Ulam-Hyers stable. The proof is completed.

Remark 4.9 A function $z \in \mathbb{E}^{1}$ is a solution of inequality (4.4) if and only if there exists a function $\Theta \in C([a, T], \mathbb{R})$ (dependent on $z$ ) such that

(i) $|\Theta(t)| \leq \varrho \Psi_{\Theta}(t), \forall t \in[a, T]$;

(ii) ${ }_{a}^{C} D^{q_{1}, \rho}\left({ }_{a}^{C} D^{q_{2}, \rho}+\lambda(t)\right) z(t)=f(t, z(t), z(\theta(t)),(\mathcal{K} z)(t))+\Theta(t), t \in[a, T]$.

By Remark 4.9, the solution of the problem

$$
{ }_{a}^{C} D^{\beta, \rho}\left({ }_{a}^{C} D^{\alpha, \rho}+\lambda(t)\right) z(t)=f(t, z(t), z(\theta(t)),(\mathcal{K} z)(t))+\Theta(t), \quad t \in[a, T],
$$

can be written as follows:

$$
\begin{aligned}
z(t)= & { }_{a}{ }^{q_{1}+q_{2}, \rho} F_{z}(s)(t)-{ }_{a} I^{q_{2}, \rho} \lambda(s) x(s)(t) \\
& +\frac{e^{\frac{\rho-1}{\rho}(t-a)}}{\Omega}\left[\left(\frac{\Omega_{4}(t-a)^{q_{2}}}{\rho^{q_{2}} \Gamma\left(q_{2}+1\right)}-\Omega_{3}\right)\right. \\
& \times\left(\sum_{j=1}^{n} \alpha_{j}\left[{ }_{a} I^{q_{1}+q_{2}+\beta_{j}, \rho} F_{x}(s)\left(\eta_{j}\right)-{ }_{a} I^{q_{2}+\beta_{j}, \rho} \lambda(s) x(s)\left(\eta_{j}\right)\right]\right. \\
& \left.-\sum_{i=1}^{m} \kappa_{i}\left[{ }_{a} I^{q_{1}+q_{2}+\mu_{i}, \rho} F_{x}(s)\left(\sigma_{i}\right)-{ }_{a} I^{q_{2}+\mu_{i}, \rho} \lambda(s) x(s)\left(\sigma_{i}\right)\right]\right) \\
& +\left(\Omega_{1}-\frac{\Omega_{2}(t-a)^{q_{2}}}{\rho^{q_{2}} \Gamma\left(q_{2}+1\right)}\right)\left(\sum_{l=1}^{r} v_{l}\left[{ }_{a} I^{q_{1}+q_{2}+\varphi_{l}, \rho} F_{x}(s)\left(\xi_{l}\right)-{ }_{a} I^{q_{2}+\varphi_{l}, \rho} \lambda(s) x(s)\left(\xi_{l}\right)\right]\right. \\
& \left.\left.-\sum_{k=1}^{p} \omega_{k}\left[I^{q_{1}+q_{2}+\gamma_{k}, \rho} F_{x}(s)\left(\psi_{k}\right)-{ }_{a} I^{q_{2}+\gamma_{k}, \rho} \lambda(s) x(s)\left(\psi_{k}\right)\right]\right)\right]+{ }_{a} I^{q_{1}+q_{2}, \rho} \Theta(s)(t) \\
& +\frac{e^{\frac{\rho-1}{\rho}(t-a)}}{\Omega}\left[\left(\frac{\Omega_{4}(t-a)^{q_{2}}}{\rho^{q_{2}} \Gamma\left(q_{2}+1\right)}-\Omega_{3}\right)\right. \\
& \times\left(\sum_{j=1}^{n} \alpha_{j a} I^{q_{1}+q_{2}+\beta_{j}, \rho} \Theta(s)\left(\eta_{j}\right)-\sum_{i=1}^{m} \kappa_{i a} I^{q_{1}+q_{2}+\mu_{i}, \rho} \Theta(s)\left(\sigma_{i}\right)\right) \\
& +\left(\Omega_{1}-\frac{\Omega_{2}(t-a)^{q_{2}}}{\rho^{q_{2}} \Gamma\left(q_{2}+1\right)}\right) \\
& \left.\times\left(\sum_{l=1}^{r} v_{l a} I^{q_{1}+q_{2}+\varphi_{l}, \rho} \Theta(s)\left(\xi_{l}\right)-\sum_{k=1}^{p} \omega_{k a} I^{q_{1}+q_{2}+\gamma_{k}, \rho} \Theta(s)\left(\psi_{k}\right)\right)\right] .
\end{aligned}
$$


Lemma 4.10 Let $z \in \mathbb{E}^{1}$ be a solution of inequality (4.4). Then the function $z$ satisfies the inequality

$$
|z(t)-(\mathcal{Q} z)(t)| \leq \Lambda_{3}\left(q_{1}+q_{2}\right) \Psi_{\Theta}(t) \varrho, \quad 0<\varrho \leq 1,
$$

where $\Lambda_{3}\left(q_{1}+q_{2}\right)$ is given by (3.4).

Proof From Remark 4.9, we obtain the inequality

$$
\begin{aligned}
\mid z(t) & -(\mathcal{Q} z)(t) \mid \\
= & \mid a^{q_{1}+q_{2}, \rho} \Psi_{\Theta}(s)(t)+\frac{e^{\frac{\rho-1}{\rho}(t-a)}}{\Omega}\left[( \frac { \Omega _ { 4 } ( t - a ) ^ { q _ { 2 } } } { \rho ^ { q _ { 2 } } \Gamma ( q _ { 2 } + 1 ) } - \Omega _ { 3 } ) \left(\sum_{j=1}^{n} \alpha_{j a} I^{q_{1}+q_{2}+\beta_{j}, \rho} \Psi_{\Theta}(s)\left(\eta_{j}\right)\right.\right. \\
& \left.-\sum_{i=1}^{m} \kappa_{i a} I^{q_{1}+q_{2}+\mu_{i}, \rho} \Psi_{\Theta}(s)\left(\sigma_{i}\right)\right) \\
& +\left(\Omega_{1}-\frac{\Omega_{2}(t-a)^{q_{2}}}{\rho^{q_{2}} \Gamma\left(q_{2}+1\right)}\right)\left(\sum_{l=1}^{r} v_{l a} I^{q_{1}+q_{2}+\varphi_{l}, \rho} \Psi_{\Theta}(s)\left(\xi_{l}\right)\right. \\
& \left.\left.-\sum_{k=1}^{p} \omega_{k a} I^{q_{1}+q_{2}+\gamma_{k}, \rho} \Psi_{\Theta}(s)\left(\psi_{k}\right)\right)\right] \mid \\
\leq & \left\{\frac{(T-a)^{q_{1}+q_{2}}}{\rho^{q_{1}+q_{2}} \Gamma\left(q_{1}+q_{2}+1\right)}\right. \\
& +\Lambda_{1}\left(\sum_{j=1}^{n} \frac{\left|\alpha_{j}\right|\left(\eta_{j}-a\right)^{q_{1}+q_{2}+\beta_{j}}}{\rho^{q_{1}+q_{2}+\beta_{j}} \Gamma\left(q_{1}+q_{2}+\beta_{j}+1\right)}+\sum_{i=1}^{m} \frac{\left|\kappa_{i}\right|\left(\sigma_{i}-a\right)^{q_{1}+q_{2}+\mu_{i}}}{\rho^{q_{1}+q_{2}+\mu_{i}} \Gamma\left(q_{1}+q_{2}+\mu_{i}+1\right)}\right) \\
& \left.+\Lambda_{2}\left(\sum_{l=1}^{r} \frac{\left|\nu_{l}\right|\left(\xi_{l}-a\right)^{q_{1}+q_{2}+\varphi_{l}}}{\rho^{q_{1}+q_{2}+\varphi_{l}} \Gamma\left(q_{1}+q_{2}+\varphi_{l}+1\right)}+\sum_{k=1}^{p} \frac{\left|\omega_{k}\right|\left(\psi_{k}-a\right)^{q_{1}+q_{2}+\gamma_{k}}}{\rho^{q_{1}+q_{2}+\gamma_{k}} \Gamma\left(q_{1}+q_{2}+\gamma_{k}+1\right)}\right)\right\} \\
& \times \Psi_{\Theta}(t) \varrho \\
= & \Lambda_{3}\left(q_{1}+q_{2}\right) \Psi_{\Theta}(t) \varrho,
\end{aligned}
$$

where $\Lambda_{1}, \Lambda_{2}, \Lambda_{3}\left(q_{1}+q_{2}\right)$ are given by (3.2), (3.3), and (3.4), respectively, which leads to the inequality in (4.9).

Next, we are ready to prove the Ulam-Hyers-Rassias and generalized Ulam-HyersRassias stability results.

Theorem 4.11 Assume that $\left(H_{1}\right),\left(H_{2}\right),\left(H_{3}\right),\left(H_{4}\right)$ are satisfied with

$$
2 L_{1} \Lambda_{3}\left(q_{1}+q_{2}\right)+L_{2} \phi_{0} \Lambda_{3}\left(q_{1}+q_{2}+1\right)+\Lambda_{4}<1 \text {. }
$$

Then problem (1.1) is both Ulam-Hyers-Rassias stable and generalized Ulam-Hyers Rassias stable on $[a, T]$. 
Proof Let $z \in \mathbb{E}^{1}$ be a solution of inequality (4.4), and let $x$ be the unique solution of problem (1.1). By applying the triangle inequality and Lemma 4.7 with (4.11), we get

$$
\begin{aligned}
& |z(t)-x(t)| \\
& =\mid z(t)-\left\{{ }_{a} I^{q_{1}+q_{2}, \rho} F_{z}(s)(t)-{ }_{a} I^{q_{2}, \rho} \lambda(s) x(s)(t)\right. \\
& +\frac{e^{\frac{\rho-1}{\rho}(t-a)}}{\Omega}\left[\left(\frac{\Omega_{4}(t-a)^{q_{2}}}{\rho^{q_{2}} \Gamma\left(q_{2}+1\right)}-\Omega_{3}\right)\right. \\
& \times\left(\sum_{j=1}^{n} \alpha_{j}\left[{ }_{a} I^{q_{1}+q_{2}+\beta_{j}, \rho} F_{x}(s)\left(\eta_{j}\right)-{ }_{a} I^{q_{2}+\beta_{j}, \rho} \lambda(s) x(s)\left(\eta_{j}\right)\right]\right. \\
& \left.-\sum_{i=1}^{m} \kappa_{i}\left[{ }_{a} I^{q_{1}+q_{2}+\mu_{i}, \rho} F_{x}(s)\left(\sigma_{i}\right)-{ }_{a} I^{q_{2}+\mu_{i}, \rho} \lambda(s) x(s)\left(\sigma_{i}\right)\right]\right) \\
& +\left(\Omega_{1}-\frac{\Omega_{2}(t-a)^{q_{2}}}{\rho^{q_{2}} \Gamma\left(q_{2}+1\right)}\right)\left(\sum_{l=1}^{r} \nu_{l}\left[a_{I^{q_{1}+q_{2}+\varphi_{l}, \rho}} F_{x}(s)\left(\xi_{l}\right)-{ }_{a} I^{q_{2}+\varphi_{l}, \rho} \lambda(s) x(s)\left(\xi_{l}\right)\right]\right. \\
& \left.\left.-\sum_{k=1}^{p} \omega_{k}\left[I_{a} I^{q_{1}+q_{2}+\gamma_{k}, \rho} F_{x}(s)\left(\psi_{k}\right)-{ }_{a} I^{q_{2}+\gamma_{k}, \rho} \lambda(s) x(s)\left(\psi_{k}\right)\right]\right)\right]+{ }_{a} I^{q_{1}+q_{2}, \rho} \Theta(s)(t) \\
& +\frac{e^{\frac{\rho-1}{\rho}(t-a)}}{\Omega}\left[\left(\frac{\Omega_{4}(t-a)^{q_{2}}}{\rho^{q_{2}} \Gamma\left(q_{2}+1\right)}-\Omega_{3}\right)\right. \\
& \times\left(\sum_{j=1}^{n} \alpha_{j a} I^{q_{1}+q_{2}+\beta_{j}, \rho} \Theta(s)\left(\eta_{j}\right)-\sum_{i=1}^{m} \kappa_{i a} I^{q_{1}+q_{2}+\mu_{i}, \rho} \Theta(s)\left(\sigma_{i}\right)\right) \\
& +\left(\Omega_{1}-\frac{\Omega_{2}(t-a)^{q_{2}}}{\rho^{q_{2}} \Gamma\left(q_{2}+1\right)}\right) \\
& \left.\left.\times\left(\sum_{l=1}^{r} v_{l a} I^{q_{1}+q_{2}+\varphi_{l}, \rho} \Theta(s)\left(\xi_{l}\right)-\sum_{k=1}^{p} \omega_{k a} I^{q_{1}+q_{2}+\gamma_{k}, \rho} \Theta(s)\left(\psi_{k}\right)\right)\right]\right\} \mid \\
& =|z(t)-(\mathcal{Q} z)(t)+(\mathcal{Q} z)(t)-(\mathcal{Q} x)(t)| \\
& \leq|z(t)-(\mathcal{Q} z)(t)|+|(\mathcal{Q} z)(t)-(\mathcal{Q} x)(t)| \\
& \leq \Lambda_{3}\left(q_{1}+q_{2}\right) \Psi_{\Theta}(t) \varrho+\left[2 L_{1} \Lambda_{3}\left(q_{1}+q_{2}\right)+L_{2} \phi_{0} \Lambda_{3}\left(q_{1}+q_{2}+1\right)+\Lambda_{4}\right]|z(t)-x(t)|,
\end{aligned}
$$

where $\Lambda_{3}(u), u=\left\{q_{1}+q_{2}, q_{1}+q_{2}+1\right\}$, and $\Lambda_{4}$ are defined by (3.4) and (3.5), respectively, which implies that

$$
|z(t)-x(t)| \leq \frac{\Lambda_{3}\left(q_{1}+q_{2}\right) \Psi_{\Theta}(t) \varrho}{1-\left[2 L_{1} \Lambda_{3}\left(q_{1}+q_{2}\right)+L_{2} \phi_{0} \Lambda_{3}\left(q_{1}+q_{2}+1\right)+\Lambda_{4}\right]} .
$$

By setting

$$
C_{f, \Phi}:=\frac{\Lambda_{3}\left(q_{1}+q_{2}\right)}{1-\left[2 L_{1} \Lambda_{3}\left(q_{1}+q_{2}\right)+L_{2} \phi_{0} \Lambda_{3}\left(q_{1}+q_{2}+1\right)+\Lambda_{4}\right]},
$$


we get the following inequality:

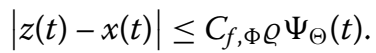

Hence, problem (1.1) is Ulam-Hyers-Rassias stable. Moreover, if we set $\Phi_{f}(t)=\varrho \Psi_{\Theta}(t)$, with $\Phi_{f}(0)=0$, then problem (1.1) is generalized Ulam-Hyers-Rassias stable. The proof is completed.

\section{Example}

In this section, we present an example which illustrates the validity and applicability of the main results.

Example 5.1 Consider the following nonlinear GPF functional integro-differential Langevin equation involving nonlocal integral conditions:

$$
\left\{\begin{array}{l}
{ }_{a}^{C} D^{\frac{3}{4}, \frac{1}{3}}\left({ }_{a}^{C} D^{\frac{1}{2}, \frac{1}{3}}+\frac{1}{25}(t-a)^{2} e^{\frac{\rho-1}{\rho}(t-a)}\right) x(t)=f(t, x(t), x(\theta(t)),(\mathcal{K} x)(t)), \quad t \in(0,2], \\
\sum_{i=1}^{3}\left(\frac{i}{3}\right)_{a} I^{\frac{i}{i+1}, \frac{1}{3}} x\left(\frac{i}{2(i+1)}\right)=\sum_{j=1}^{2}\left(\frac{j}{4}\right)_{a} I^{j+1}, \frac{1}{3} x\left(\frac{j}{j+6}\right), \\
\sum_{k=1}^{2}\left(\frac{k}{5}\right)_{a} I^{\frac{k+2}{k+3}, \frac{1}{3}} x\left(\frac{\sqrt{k}}{k^{2}+2}\right)=\sum_{l=1}^{3}\left(\frac{l}{6}\right)_{a} I^{l+3}, \frac{1}{3} x\left(\frac{2 l}{3 l+2}\right) .
\end{array}\right.
$$

Here, $q_{1}=\frac{1}{2}, q_{2}=\frac{1}{2}, \rho=\frac{1}{3}, a=0, T=2, m=3, n=2, p=2, r=3, \kappa_{i}=\frac{i}{3}, \sigma_{i}=\frac{i}{2(i+1)}, \mu_{i}=$ $\frac{i}{i+1}, i=1,2,3, \alpha_{j}=\frac{j}{4}, \eta_{j}=\frac{j}{j+6}, \beta_{j}=\frac{j+1}{j+2}, j=1,2, \omega_{k}=\frac{k}{5}, \psi_{j}=\frac{\sqrt{k}}{k^{2}+2}, \gamma_{k}=\frac{k+2}{k+3}, k=1,2, v_{l}=\frac{l}{6}$, $\xi_{l}=\frac{2 l}{3 l+2}, \varphi_{l}=\frac{l+3}{l+4}, l=1,2,3, \theta(t)=\frac{t}{2}$, and

$$
\lambda(t)=\frac{1}{25}(t-a)^{2} e^{\frac{\rho-1}{\rho}(t-a)} .
$$

Obviously, the function $\lambda$ satisfies $\left(H_{2}\right)$ for all $t \in[a, T]$. From the given data, we obtain that $\Omega_{1} \approx 0.6995071719, \Omega_{2} \approx 0.7639237899, \Omega_{3} \approx-0.3023660189, \Omega_{4} \approx-0.2312067168$, $\Omega \approx 0.0662301783 \neq 0$. Furthermore, we assume the nonlinearity as follows:

(i) Let $f:[a, T] \times \mathbb{R}^{3} \rightarrow \mathbb{R}$ be a function, which is given by

$$
\begin{aligned}
& f(t, x(t), x(\theta(t)),(\mathcal{K} x)(t)) \\
& \quad=\frac{1}{2}+\frac{t^{2}}{3}+\frac{2 \cos ^{2}(\pi t)}{(t+16)^{2}} \frac{|x|}{1+|x|}-\frac{x(0.5 t)}{(t+16)^{2}}+\frac{(t+2)^{3}}{\left(8 e^{t}+1\right)^{2}} \int_{a}^{t} \frac{\cos ^{2}(\pi t)}{\left(e^{s^{2}}+1\right)^{2}} x(s) d s .
\end{aligned}
$$

For $x_{1}, x_{2}, y_{1}, y_{2}, z_{1}, z_{2} \in \mathbb{R}$ and $t \in[a, T]$, we have

$$
\begin{aligned}
& \left|f\left(t, x_{1}, y_{1}, z_{1}\right)-f\left(t, x_{2}, y_{2}, z_{2}\right)\right| \leq \frac{1}{(t+16)^{2}}\left(\left|x_{1}-y_{1}\right|+\left|x_{2}-y_{2}\right|\right)+\frac{(t+2)^{3}}{\left(8 e^{t}+1\right)^{2}}\left|z_{1}-z_{2}\right|, \\
& \left|\phi\left(t, s, x_{1}\right)-\phi\left(t, s, y_{1}\right)\right| \leq \frac{1}{4}\left|x_{1}-y_{1}\right| .
\end{aligned}
$$

Hypotheses $\left(H_{1}\right)-\left(H_{4}\right)$ are satisfied with $L_{1}=\frac{1}{256}, L_{2}=\frac{1}{81}$, and $\phi_{0}=\frac{1}{4}$. Hence

$$
2 L_{1} \Lambda_{3}\left(q_{1}+q_{2}\right)+L_{2} \phi_{0} \Lambda_{3}\left(q_{1}+q_{2}+1\right)+\Lambda_{4} \approx 0.7833485782<1 .
$$


Since all the hypotheses of Theorem 3.1 are satisfied, problem (5.1) has a unique solution on $[0,2]$. Moreover, we can also compute that

$$
\Phi:=\frac{\Lambda_{3}\left(q_{1}+q_{2}\right)}{1-\left[2 L_{1} \Lambda_{3}\left(q_{1}+q_{2}\right)+L_{2} \phi_{0} \Lambda_{3}\left(q_{1}+q_{2}+1\right)+\Lambda_{4}\right]} \approx 375.8602857>0 .
$$

Hence, by Theorem 4.8, problem (5.1) is both Ulam-Hyers and also generalized UlamHyers stable.

(ii) Let $f:[a, T] \times \mathbb{R}^{3} \rightarrow \mathbb{R}$ be a function which is given by

$$
\begin{aligned}
f(t, x(t), x(\theta(t)),(\mathcal{K} x)(t))= & \frac{2 e^{t}}{(t+1)^{2}}+\frac{e^{-t}}{2(t+9)^{2}} \cdot \frac{|x|}{2+|x|} \\
& +\frac{1}{(t+9)^{2}} \cdot \frac{|x(0.75 t)|}{|x(0.75 t)|+4}+\frac{\sin ^{2}(\pi t)}{e^{t}+1} \int_{a}^{t} \frac{\cos ^{2}(t-s)}{\left(e^{t-s}+1\right)^{2}} x(s) d s .
\end{aligned}
$$

It is easy to see that, for all $x_{1}, x_{2}, y_{1}, y_{2}, z_{1}, z_{2} \in \mathbb{R}$ and $t \in[a, T]$, we get

$$
\begin{aligned}
&\left|f\left(t, x_{1}, y_{1}, z_{1}\right)-f\left(t, x_{2}, y_{2}, z_{2}\right)\right| \leq \frac{1}{4(t+9)^{2}}\left(\left|x_{1}-y_{1}\right|+\left|x_{2}-y_{2}\right|\right) \\
&+\frac{1}{\left(e^{t}+1\right)^{3}}\left|z_{1}-z_{2}\right| \\
&\left|\phi\left(t, s, x_{1}\right)-\phi\left(t, s, y_{1}\right)\right| \leq \frac{1}{16}\left|x_{1}-y_{1}\right| .
\end{aligned}
$$

Hypotheses $\left(H_{1}\right)-\left(H_{4}\right)$ are satisfied with $L_{1}=\frac{1}{324}, L_{2}=\frac{1}{8}$, and $\phi_{0}=\frac{1}{16}$. Hence

$$
2 L_{1} \Lambda_{3}\left(q_{1}+q_{2}\right)+L_{2} \phi_{0} \Lambda_{3}\left(q_{1}+q_{2}+1\right)+\Lambda_{4} \approx 0.7348101092<1 .
$$

Furthermore, for $x, y, z \in \mathbb{R}$ and $t \in[a, T]$, it follows that

$$
|f(t, x, y, z)| \leq \frac{2 e^{t}}{(t+1)^{2}}+\frac{e^{-t}}{4(t+9)^{2}}|x|+\frac{1}{4(t+9)^{2}}|y|+\frac{1}{\left(e^{t}+1\right)^{3}}|z| .
$$

Hypothesis $\left(H_{5}\right)$ is also valid with $h_{1}(t)=\frac{2 e^{t}}{(t+1)^{2}}, h_{2}(t)=\frac{e^{-t}}{4(t+9)^{2}}, h_{3}(t)=\frac{1}{4(t+9)^{2}}, h_{4}(t)=\frac{1}{\left(e^{t}+1\right)^{3}}$, and $h_{1}^{*}=2, h_{2}^{*}=h_{3}^{*}=\frac{1}{324}, h_{4}^{*}=\frac{1}{8}$. Therefore, all the hypotheses of Theorem 3.3 are fulfilled, which concludes that problem (5.1) has at least one solution on [0,2]. Moreover, we obtain

$$
C_{f, \Phi}:=\frac{\Lambda_{3}\left(q_{1}+q_{2}\right)}{1-\left[2 L_{1} \Lambda_{3}\left(q_{1}+q_{2}\right)+L_{2} \phi_{0} \Lambda_{3}\left(q_{1}+q_{2}+1\right)+\Lambda_{4}\right]} \approx 307.0654958>0 .
$$

Hence, by Theorem 4.11, problem (5.1) is both Ulam-Hyers-Rassias and generalized Ulam-Hyers-Rassias stable.

\section{Conclusion}

Langevin equation is an important equation of mathematical physics that is used in modeling the phenomena occurring in fluctuating environment such as Brownian motion. In the literature, it is also referred to as a stochastic differential equation as it governs the fast motion of microscopic variables of the dynamical systems. It has been realized, however, that the integer order Langevin equation cannot provide elaborate description to 
the complex systems that involve disordered or fractal medium. Therefore, attention toward considering noninteger order Langevin equation becomes urgent and compulsory. Thus, the boundary value problems defined by fractional Langevin equation have been extensively studied in recent years. Based on their interests and demands, the authors have considered Langevin equation within different types of fractional derivatives and boundary conditions. The fractional derivatives have been often utilized in the frame of Caputo, Riemann-Liouville, or Hadamard settings, whereas the supplemented boundary conditions have been of nonlocal, anti periodic, or mixed types.

In this paper, we study new Langevin equation within the so-called GPF derivative. Fixed point theorems and Ulam's approach are employed to investigate the existence, uniqueness, and different types of stability. The results of this paper not only generalize previous results but also provide a totally different approach in the sense that different fractional derivative is accommodated, different boundary conditions are associated, different fixed point theorems are used, and Ulam stability within GPF derivative is discussed. We believe that the results of this paper will provide considerable potential to interested researchers to produce relevant results concerning qualitative properties of nonlinear GPF differential equations.

\begin{abstract}
Acknowledgements
The first author was supported by the International Science Programme Ph.D. Research Scholarship from National University of Laos (NUOL) and Burapha University. The second author would like to thank for funding this work through the Center of Excellence in Mathematics (CEM), CHE, Sri Ayutthaya Rd., Bangkok, 10400, Thailand and Barapha University. J. Alzabut would like to thank Prince Sultan University for funding this work through research group Nonlinear Analysis Methods in Applied Mathematics (NAMAM) group number RG-DES-2017-01-17, and the fourth author was financially supported by Navamindradhiraj University through the Navamindradhiraj University Research Fund (NURF). Moreover, the authors would like to thank the referees for their careful reading of the article and insightful comments.
\end{abstract}

Funding

Not applicable.

Availability of data and materials

The authors declare that all data and materials in this paper are available and veritable.

Competing interests

The authors declare that they have no competing interests.

Authors' contributions

This is to declare that all authors have contributed equally and significantly to the contents of the paper. All authors have read and agreed to the published version of the manuscript.

\title{
Author details
}

${ }^{1}$ Department of Mathematics, Faculty of Science, Burapha University, Chonburi, 22000, Thailand. ${ }^{2}$ Department of Mathematics and General Sciences, Prince Sultan University, Riyadh, 11586, Saudi Arabia. ${ }^{3}$ Department of General Education, Faculty of Science and Health Technology, Navamindradhiraj University, Bangkok, 10300, Thailand.

\section{Publisher's Note}

Springer Nature remains neutral with regard to jurisdictional claims in published maps and institutional affiliations.

Received: 18 May 2020 Accepted: 30 November 2020 Published online: 09 December 2020

References

1. Podlubny, I.: Fractional Differential Equations. Academic Press, New York (1999)

2. Hilfer, R.: Applications of Fractional Calculus in Physics. World Scientific, Singapore (2000)

3. Kilbas, A.A., Srivastava, H.M., Trujillo, J.J.: Theory and Applications of Fractional Differential Equations. North-Holland Mathematics Studies, vol. 204. Elsevier, Amsterdam (2006)

4. Magin, R.: Fractional Calculus in Bioengineering. Begall House Publisher, Inc., Connecticut (2006)

5. Mainardi, F.: Fractional Calculus and Waves in Linear Viscoelasticity: An Introduction to Mathematical Models. Imperial College Press, Singapore (2010)

6. Wang, G.: Twin iterative positive solutions of fractional q-difference Schrödinger equations. Appl. Math. Lett. 76 103-109 (2018) 
7. Zhang, L., Ahmad, B., Wang, G., Ren, X.: Radial symmetry of solution for fractional p-Laplacian system. Nonlinear Anal. $196,111801(2020)$

8. Wang, G., Ren, X.: Radial symmetry of standing waves for nonlinear fractional Laplacian Hardy-Schrödinger systems. Appl. Math. Lett. 110, 106560 (2020)

9. Zhang, L., Hou, W.: Standing waves of nonlinear fractional p-Laplacian Schrödinger equation involving logarithmic nonlinearity. Appl. Math. Lett. 102, 106149 (2020)

10. Langevin, P.: On the theory of Brownian motion. C. R. Acad. Bulgare Sci. 1908(10), 140-154 (1908)

11. Kubo, R.: The fluctuation-dissipation theorem. Rep. Prog. Phys. 29, 255-284 (1966)

12. Mainardi, F., Pironi, P.: The fractional Langevin equation: Brownian motion revised. Extr. Math. 1996(10), 140-154 (1996)

13. Wax, N.: Selected Papers on Noise and Stochastic Processes. Dover, New York (1954)

14. Mazo, R.: Brownian Motion: Fluctuations, Dynamics and Applications. Oxford Univ. Press, Oxford (2002)

15. Coffey, W.T., Kalmykov, Y.P., Waldron, J.T.: The Langevin Equation with Applications to Stochastic Problems in Physics, Chemistry and Electrical Engineering, 2nd edn. World Scientific, Singapore (2004)

16. Mainardi, F., Pironi, P., Tampieri, F.: On a generalized of the Basset problem via fractional calculus. Proc. CANCAM 95(2), 836-837 (1995)

17. Lim, S.C., Li, M., Teo, L.P.: Langevin equation with two fractional orders. Phys. Lett. A 372(42), 6309-6320 (2008)

18. Ahmad, B., Nieto, J.J: Solvability of nonlinear Langevin equation involving two fractional orders with Dirichlet boundary conditions. Int. J. Differ. Equ. 2010, Article ID 649486 (2010)

19. Ahmad, B., Nieto, J.J., Alsaedi, A., El-Shahed, M.: A study of nonlinear Langevin equation involving two fractional orders in different intervals. Nonlinear Anal., Real World Appl. 13(2), 599-606 (2012)

20. Wang, G., Zhang, L., Song, G.: Boundary value problem of a nonlinear Langevin equation with two different fractional orders and impulses. Fixed Point Theory Appl. 2012(1), 200 (2012)

21. Sudsutad, W., Tariboon, J.: Nonlinear fractional integro-differential Langevin involving two fractional orders with three-point multi-term fractional integral boundary conditions. J. Appl. Math. Comput. 43(1-2), 507-522 (2013)

22. Tariboon, J., Ntouyas, S.K., Thaiprayoon, C.: Nonlinear Langevin equation of Hadamard Caputo type fractional derivatives with nonlocal fractional integral conditions. Adv. Math. Phys. 2014, Article ID 372749 (2014)

23. Thaiprayoon, C., Ntouyas, S.K., Tariboon, J.: On the nonlocal Katugampola fractional integral conditions for fractional Langevin equation. Adv. Differ. Equ. 2015, 374 (2015)

24. Zhou, H., Alzabut, J., Yang, L.: On fractional Langevin differential equations with anti-periodic boundary conditions. Eur. Phys. J. Spec. Top. 226(16-18), 3577-3590 (2017)

25. Baghani, H.: Existence and uniqueness of solutions to fractional Langevin equations involving two fractional orders. J. Fixed Point Theory Appl. 2018(20), 63 (2018)

26. Fazli, H., Nieto, J.J.: Fractional Langevin equations with anti periodic boundary conditions. Chaos Solitons Fractals 114, 332-337 (2018)

27. Berhail, A., Tabouche, N., Matar, M.M., Alzabut, J.: On nonlocal integral and derivative boundary value problem of nonlinear Hadamard Langevin equation with three different fractional orders. Bol. Soc. Mat. Mex. 26, 303-318 (2019)

28. Ahmad, B., Alsaedi, A., Ntouyas, S.K.: Nonlinear Langevin equations and inclusions involving mixed fractional order derivatives and variable coefficient with fractional nonlocal terminal conditions. AIMS Math. 4(3), 626-647 (2019)

29. Wang, G., Qin, J., Zhang, L., Baleanu, D.: Explicit iteration to a nonlinear fractional Langevin equation with non-separated integro-differential strip-multi-point boundary conditions. Chaos Solitons Fractals 131, 109476 (2019)

30. Baleanu, D., Darzi, R., Agheli, B.: Existence results for Langevin equation involving Atangana-Baleanu fractional operators. Mathematics 8, 408, 1-12 (2020)

31. Wongcharoen, A., Ahmad, B., Ntouyas, S.K., Tariboon, J.: Three-point boundary value problems for the Langevin equation with the Hilfer fractional derivative. Adv. Math. Phys. 2020, Article ID 9606428 (2020)

32. Alzabut, J., Abdeljawad, T., Jarad, F., Sudsutad, W.: A Gronwall inequality via the generalized proportional fractional derivative with applications. J. Inequal. Appl. 2019, 101 (2019)

33. Abdeljawad, T., Jarad, F., Mallak, S., Alzabut, J.: Lyapunov type inequalities via fractional proportional derivatives and application on the free zero disc of Kilbas-Saigo generalized Mittag-Leffler functions. Eur. Phys. J. Plus 134, 247 (2019)

34. Alzabut, J., Sudsutad, W., Kayar, Z., Baghani, H.: A new Gronwall-Bellman inequality in a frame of generalized proportional fractional proportional derivative. Mathematics 7, 747, 1-15 (2019)

35. Sudsutad, W., Alzabut, J., Tearnbucha, C., Thaiprayoon, C.: On the oscillation of differential equations in frame of generalized proportional fractional derivatives. AIMS Math. 5(2), 856-871 (2020)

36. Sudsutad, W., Alzabut, J., Nontasawatsri, S., Thaiprayoon, C.: Stability analysis for a generalized proportional fractional Langevin equation with variable coefficient and mixed integro-differential boundary conditions. J. Nonlinear Funct. Anal. 23, 1-24 (2020)

37. Alzabut, J., Viji, J., Muthulakshmi, V., Sudsutad, W.: Oscillatory behavior of a type of generalized proportional fractional differential equations with forcing and damping terms. Mathematics 8, 1037, 1-18 (2020)

38. Jarad, J., Abdeljawad, T., Alzabut, J.: Generalized fractional derivatives generated by a class of local proportional derivatives. Eur. Phys. J. Spec. Top. 226, 3457-3471 (2017)

39. Rus, I.A.: Ulam stabilities of ordinary differential equations in a Banach space. Carpath. J. Math. 26, 103-107 (2010)

40. Granas, A., Dugundji, J.: Fixed Point Theory. Springer, New York (2003)

41. Krasnoselskii, M.A.: Two remarks on the method of successive approximations. Usp. Mat. Nauk 10, 123-127 (1955) 